\title{
An angular momentum approach to quadratic Fourier transform, Hadamard matrices, Gauss sums, mutually unbiased bases, unitary group and Pauli group 1
}

\author{
Maurice R Kibler \\ Université de Lyon, F-69622, Lyon, France \\ Université Lyon 1, Villeurbanne, France \\ and \\ CNRS/IN2P3, Institut de Physique Nucléaire de Lyon, France \\ E-mail: m.kibler@ipnl.in2p3.fr
}

\begin{abstract}
The construction of unitary operator bases in a finite-dimensional Hilbert space is reviewed through a nonstandard approach combinining angular momentum theory and representation theory of $S U(2)$. A single formula for the bases is obtained from a polar decomposition of $S U(2)$ and analysed in terms of cyclic groups, quadratic Fourier transforms, Hadamard matrices and generalized Gauss sums. Weyl pairs, generalized Pauli operators and their application to the unitary group and the Pauli group naturally arise in this approach.

PACS numbers: 03.65.Fd, 03.65.Ta, 03.65.Ud, 02.20.Qs

Keywords: finite quantum mechanics - angular momentum - Weyl pairs - generalized Pauli operators - quadratic Fourier transform - Hadamard matrices - Gauss sums - mutually unbiased bases - cyclic group - unitary group - Heisenberg-Weyl group - Pauli group
\end{abstract}

\footnotetext{
${ }^{1}$ Dedicated to the memory of Yurii Fedorovich Smirnov.
} 


\section{Introduction}

Angular momentum theory [1] and its group-theoretical formulation in terms of the WignerRacah algebra of $S U(2)$ [2, 3, 4] (see also [5] for an extension to a finite or compact group) are of central importance in subatomic, atomic, molecular and condensed matter physics. The components of any angular momentum (spin, isopin, orbital angular momentum, etc.) generate the Lie algebra of the group $S U(2)$. Therefore, $S U(2)$ and its noncompact extension $S U(1,1)$ are basic ingredients for dealing with generalized angular momenta. Chains of groups ending with $S O(3) \simeq S U(2) / \mathbb{Z}_{2}(+)$ or $S O(3) \subset S O(2)$ are of interest in subatomic and atomic physics. In this direction, one can mention the group $S U(3) \otimes S U(2) \otimes U(1)$ (related to the chain $U(3) \subset S U(2) \otimes U(1) \subset U(1)$ ) and its grand unified and/or supersymmetric extensions for describing elementary particles and their (strong and electroweak) interactions [6]. Furthermore, one know the relevance in atomic physics of the chain $U(7) \subset S O(7) \subset G_{2} \subset S O(3) \subset S O(2)$ for the electronic spectroscopy of $f^{N}$ ions [3]. On the other side, chains ending with $S U(2) \subset G$, where $G$ is a finite group (or a chain involving finite groups), proved to be of considerable interest in molecular and condensed matter spectroscopy [7, 8, 9]. Recently, chains of type $S U(2) \subset G$ were also used in attempts to understand the flavor structure of quarks and leptons [10]. The groups $S U(2)$ and $S U(1,1)$, as well as their $q$ - or $q p$-deformations in the sense of Hopf algebras (see for instance [11, 12], thus play a pivotal role in many areas of physical sciences.

The representation theory of $S U(2)$ is generally adressed in two different ways. The standard one amounts to diagonalise the complete set $\left\{j^{2}, j_{z}\right\}$ involving the Casimir operator $j^{2}$ and one generator $j_{z}$ of $S U(2)$. Another way is to consider a set $\left\{j^{2}, v\right\}$, where $v$ is an operator defined in the enveloping algebra of $S U(2)$ and invariant under a subgroup of $S U(2)$. A third way (not very well-known) consists in diagonalising a complete set $\left\{j^{2}, v_{r a}\right\}$, where $v_{r a}$ stands for a two-parameter operator which commutes with $j^{2}$ and is a pseudoinvariant under a cyclic group [13].

It is the aim of this review paper to show that the third approach to the representation theory of $S U(2)$ opens a window on the apparently disconnected subjects enumerated in the title.

The plan of the paper is as follows. The minimal requirements for a $\left\{j^{2}, v_{r a}\right\}$ approach to $S U(2)$ (i.e., a nonstandard approach to angular momentum theory) are given in Section 2 and in two appendices. Section 3 deals with quadratic sums (in relation with quadratic discrete Fourier transforms, generalized Hadamard matrices, generalized quadratic Gauss sums and mutually unbiased bases) and Section 4 is devoted to unitary groups and Pauli groups. 
The present paper is dedicated to the memory of the late Professor Yurii Fedorovich Smirnov who contributed to many domains of mathematical physics (e.g., Lie groups and Lie algebras, quantum groups, special functions) and theoretical physics (e.g., nuclear, atomic and molecular physics, crystal- and ligand-field theory).

A few words about some of the notations is in order. The bar indicates complex conjugation. The symbol $\delta_{a, b}$ stands for the Kronecker symbol of $a$ and $b$. We use $I$ and $I_{d}$ to denote the identity operator and the $d$-dimensional unity matrix, respectively. The operator $A^{\dagger}$ stands for the adjoint of the operator $A$. We note as $[A, B]_{-}$and $[A, B]_{+}$ the commutator and the anticommutator of the operators $A$ and $B$, respectively. We use the Dirac notation $|\psi\rangle$ for a vector in an Hilbert space; furthermore, $\langle\phi \mid \psi\rangle$ and $|\phi\rangle\langle\psi|$ are respectively the inner product and the outer product of the vectors $|\psi\rangle$ and $|\phi\rangle$. The symbols $\oplus$ and $\ominus$ stand respectively for the addition and subtraction modulo $d$ while $\otimes$ and $\uplus$ are used respectively for the direct product of vectors or operators and the direct sum of vector spaces. The matrices of type $E_{\lambda, \mu}$ with the matrix elements

$$
\left(E_{\lambda, \mu}\right)_{\lambda^{\prime}, \mu^{\prime}}:=\delta_{\lambda, \lambda^{\prime}} \delta_{\mu, \mu^{\prime}}
$$

stand for generators of the Lie group $G L(d, \mathbb{C})$. For $a$ and $b$ coprime, we take

$$
\left(\begin{array}{l}
a \\
b
\end{array}\right)_{L}:=\left\{\begin{array}{l}
+1 \text { if } a=k^{2} \bmod (b) \\
-1 \text { si } a \neq k^{2} \bmod (b)
\end{array}\right.
$$

to denote the Legendre symbol of $a$ and $b$ (equal to 1 if $a$ is a quadratic residu modulo $b$ and -1 if $a$ is not a quadratic residu modulo $b$ ). In addition, the integer inverse $(a \backslash b)$ of $a$ with respect to $b$ is given by

$$
a(a \backslash b)=1 \bmod (b)
$$

Finally, the $q$-deformed number $[n]_{q}$ and the $q$-deformed factorial $[n]_{q} !$, with $n \in \mathbb{N}$, are defined by

$$
[n]_{q}:=\frac{1-q^{n}}{1-q}
$$

and

$$
[n]_{q} !:=[1]_{q}[2]_{q} \ldots[n]_{q} \quad[0]_{q} !:=1
$$

where $q$ is taken is this paper as a primitive root of unity. 


\section{A nonstandard approach to $s u(2)$}

In some previous works [13], we developed a nonstandard approach to the Lie algebra $s u(2)$ and studied the corresponding Wigner-Racah algebra of the group $S U(2)$. This nonstandard approach is based on a polar decomposition of $s u(2)$, based in turn on a troncated oscillator algebra (see Appendices A and B). It yields nonstandard bases for the irreducible representations of $S U(2)$ and new Clebsch-Gordan coefficients for the angular momentum theory. Basically, the approach amounts to replace the set $\left\{j^{2}, j_{z}\right\}$, familiar in quantum mechanics, by a set $\left\{j^{2}, v_{r a}\right\}\left(j^{2}\right.$ and $j_{z}$ are the Casimir operator and the Cartan generator of $s u(2)$, respectively).

The operator $v_{r a}$ acts on the $(2 j+1)$-dimensional subspace $\mathcal{E}(2 j+1)$, associated with the angular momentum $j$, of the representation space of $S U(2)$. We define it here by

$$
v_{r a}:=e^{i 2 \pi j r}|j,-j\rangle\left\langle j, j\left|+\sum_{m=-j}^{j-1} q^{(j-m) a}\right| j, m+1\right\rangle\langle j, m|
$$

where

$$
q:=\exp \left(\frac{2 \pi i}{2 j+1}\right) \quad 2 j \in \mathbb{N} \quad r \in \mathbb{R} \quad a \in \mathbb{Z}_{2 j+1}
$$

and, for fixed $j$, the vectors $|j, m\rangle$ (with $m=j, j-1, \ldots,-j$ ) satisfy the eigenvalue equations

$$
j^{2}|j, m\rangle=j(j+1)|j, m\rangle \quad j_{z}|j, m\rangle=m|j, m\rangle
$$

familiar in angular momentum theory. The vectors $|j, m\rangle$ span the Hilbert space $\mathcal{E}(2 j+$ 1) $\sim \mathbb{C}^{2 j+1}$ and are taken in an orthonormalized form with

$$
\left\langle j, m \mid j, m^{\prime}\right\rangle=\delta_{m, m^{\prime}} .
$$

Obviously, the operator $v_{r a}$ is unitary and commutes with $j^{2}$. The spectrum of the set $\left\{j^{2}, v_{r a}\right\}$ is described by

Result 1. For fixed $j, r$ and $a$, the $2 j+1$ vectors

$$
|j \alpha ; r a\rangle:=\frac{1}{\sqrt{2 j+1}} \sum_{m=-j}^{j} q^{(j+m)(j-m+1) a / 2-j m r+(j+m) \alpha}|j, m\rangle
$$

with $\alpha=0,1, \ldots, 2 j$, are common eigenvectors of $v_{r a}$ and $j^{2}$. The eigenvalues of $v_{r a}$ and $j^{2}$ are given by

$$
v_{r a}|j \alpha ; r a\rangle=q^{j(a+r)-\alpha}|j \alpha ; r a\rangle \quad j^{2}|j \alpha ; r a\rangle=j(j+1)|j \alpha ; r a\rangle \quad \alpha=0,1, \ldots, 2 j .
$$


The spectrum of $v_{\text {ra }}$ is nondegenerate.

The set $\{|j \alpha ; r a\rangle: \alpha=0,1, \ldots, 2 j\}$ constitutes another orthonormal basis, besides the basis $\{|j, m\rangle: m=j, j-1, \ldots,-j\}$, of $\mathcal{E}(2 j+1)$ in view of

$$
\langle j \alpha ; r a \mid j \beta ; r a\rangle=\delta_{\alpha, \beta} .
$$

Note that the value of $\langle j \alpha ; r a \mid j \beta ; s b\rangle$ is much more involved for $r \neq s$ and $a \neq b$ and needs the calculation of Gauss sums as we shall see below.

The Wigner-Racah algebra of $S U(2)$ can be developed in the $\left\{j^{2}, v_{r a}\right\}$ scheme. This leads to Clebsch-Gordan coefficients and $(3-j \alpha)_{r a}$ symbols with properties very different from the ones of the usual $S U(2) \subset U(1)$ Clebsch-Gordan coefficients and $3-j m$ symbols corresponding to the $\left\{j^{2}, j_{z}\right\}$ scheme [13].

The nonstandard approach to angular momentum theory briefly summarized above is especially useful in quantum chemistry for problems involving cyclic symmetry. This is the case for a ring-shape molecule with $2 j+1$ atoms at the vertices of a regular polygon with $2 j+1$ sides or for a one-dimensional chain of $2 j+1$ spins ( $\frac{1}{2}$-spin each) [14]. In this connection, we observe that the vectors of type $|j \alpha ; r a\rangle$ are specific symmetryadapted vectors [15, 16]. Symmetry-adapted vectors are widely used in quantum chemistry, molecular physics and condensed matter physics as for instance in rotational spectroscopy of molecules [17] and ligand-field theory [18]. However, the vectors $|j \alpha ; r a\rangle$ differ from the symmetry-adapted vectors considered in Refs. [19, 20, 21, 22] in the sense that $v_{r a}$ is not an invariant under some finite subgroup (of crystallographic interest) of the orthogonal group $O(3)$. Indeed, $v_{r a}$ is a pseudoinvariant [23] under the Wigner operator $P_{R(\varphi)}$ associated with the rotation $R(\varphi)$, around the quantization axis $O z$, with the angle

$$
\varphi:=p \frac{2 \pi}{2 j+1} \quad p=0,1, \ldots, 2 j
$$

since

$$
P_{R(\varphi)} v_{r a} P_{R(\varphi)}^{\dagger}=e^{-i \varphi} v_{r a}
$$

More precisely, we have

Result 2. The operator $v_{\text {ra }}$ transforms according to an irreducible representation of the cyclic subgroup $C_{2 j+1} \sim \mathbb{Z}_{2 j+1}(+)$ of the special orthogonal group $S O(3)$. In terms of vectors, one has

$$
P_{R(\varphi)}|j \alpha ; r a\rangle=q^{j p}|j \beta ; r a\rangle \quad \beta:=\alpha \ominus p
$$

so that the set $\{|j \alpha ; r a\rangle: \alpha=0,1, \ldots, 2 j\}$ is stable under $P_{R(\varphi)}$. The latter set spans the regular representation of $C_{2 j+1}$. 


\section{Variations on quadratic sums}

\subsection{Quadratic discrete Fourier transform}

We leave the domain of angular momentum theory and adopt the following notations

$$
d:=2 j+1 \quad k:=j-m \quad|k\rangle:=|j, m\rangle .
$$

These notations are particularly adapted to quantum information and quantum computation. In these new notations, we have

$$
v_{r a}=e^{i \pi(d-1) r}|d-1\rangle\left\langle 0\left|+\sum_{k=1}^{d-1} q^{k a}\right| k-1\right\rangle\langle k| .
$$

From now on, we assume that $d \geq 2$ and $r=0$ (the case $d=1$ and $r \neq 0$, although of interest in the theory of angular momentum, is not essential for what follows). In addition, we put

$$
|a \alpha\rangle:=|j \alpha ; 0 a\rangle
$$

with $a$ and $\alpha$ in the ring $\mathbb{Z}_{d}:=\mathbb{Z} / d \mathbb{Z}$. Then, Eq. (11) gives

$$
v_{0 a}|a \alpha\rangle=q^{(d-1) a / 2-\alpha}|a \alpha\rangle
$$

with

$$
|a \alpha\rangle=\frac{1}{\sqrt{d}} \sum_{k=0}^{d-1} q^{(k+1)(d-k-1) a / 2-(k+1) \alpha}|k\rangle .
$$

Equation (20) can be rewritten as

$$
|a \alpha\rangle=\sum_{k=0}^{d-1}\left(F_{a}\right)_{k \alpha}|k\rangle
$$

where

$$
\left(F_{a}\right)_{k \alpha}:=\frac{1}{\sqrt{d}} q^{(k+1)(d-k-1) a / 2-(k+1) \alpha}
$$

is the $k \alpha$-th matrix element of a $d \times d$ matrix $F_{a}$ (the matrix $F_{a}$ can be seen as the matrix associated with the character table of the cyclic group $C_{d}$ pre- and post-multiplied by diagonal matrices). 
Equations (21)-(22) define a quadratic quantum Fourier transform. The matrix $F_{a}$ is unitary so that (21) can be inverted to give

$$
|k\rangle=\sum_{\alpha=0}^{d-1} \overline{\left(F_{a}\right)_{k \alpha}}|a \alpha\rangle
$$

or

$$
|k\rangle=\frac{1}{\sqrt{d}} \sum_{\alpha=0}^{d-1} q^{-(k+1)(d-k-1) a / 2+(k+1) \alpha}|a \alpha\rangle .
$$

In the special case $a=0$, we have

$$
|0 \alpha\rangle=q^{-\alpha} \frac{1}{\sqrt{d}} \sum_{k=0}^{d-1} e^{-\frac{2 \pi i}{d} \alpha k}|k\rangle \Leftrightarrow|k\rangle=\frac{1}{\sqrt{d}} \sum_{\alpha=0}^{d-1} e^{\frac{2 \pi i}{d}(k+1) \alpha}|0 \alpha\rangle .
$$

Consequently, the quadratic quantum Fourier transform reduces to the ordinary quantum Fourier transform (up to a phase factor). The corresponding matrix $F_{0}$ satisfies

$$
F_{0}^{4}=q I_{d}
$$

to be compared to the well-known relation $F^{4}=I_{d}$ for the standard quantum Fourier transform [24].

At this stage, we forsee that $d+1$ (orthonormal) bases of the space $\mathcal{E}(d)$ play an important role in the present paper: (i) the basis

$$
B_{d}:=\{|j, m\rangle: m=j, j-1, \ldots,-j\} \Leftrightarrow B_{d}:=\{|k\rangle: k=0,1, \ldots, d-1\}
$$

associated with the $\left\{j^{2}, j_{z}\right\}$ scheme, known as the spherical or canonical basis in the theory of angular momentum, and as the computational basis in quantum information and quantum computation and (ii) the $d$ bases

$$
B_{a}:=\{|a \alpha\rangle: \alpha=0,1, \ldots, d-1\} \quad a=0,1, \ldots, d-1
$$

(noted $B_{0 a}$ in Ref. [25]) associated with the $\left\{j^{2}, v_{0 a}\right\}$ scheme.

To close this subsection, let us show how the preceding developments can be used for defining a quadratic discrete Fourier transform. We start from the formal transformation

$$
x:=\{x(k) \in \mathbb{C}: k=0,1, \ldots, d-1\} \rightarrow y:=\{y(\alpha) \in \mathbb{C}: \alpha=0,1, \ldots, d-1\}
$$

defined via

$$
y(\alpha):=\frac{1}{\sqrt{d}} \sum_{k=0}^{d-1} q^{(k+1)(d-k-1) a / 2-(k+1) \alpha} x(k)
$$


where $a$ can take any of the values $0,1, \ldots, d-1$. Alternatively, for fixed $a$ we have

$$
y(\alpha)=\sum_{k=0}^{d-1}\left(F_{a}\right)_{k \alpha} x(k) \quad \alpha=0,1, \ldots, d-1 .
$$

The inverse transformation $y \rightarrow x$ is described by

$$
x(k)=\sum_{\alpha=0}^{d-1} \overline{\left(F_{a}\right)_{k \alpha}} y(\alpha) \quad k=0,1, \ldots, d-1 .
$$

The bijective transformation $x \leftrightarrow y$ can be thought of as a quadratic discrete Fourier transform. The case $a=0$ corresponds to the ordinary discrete Fourier transform (up to a phase factor). These matters lead to the following result which generalizes the ParsevalPlancherel theorem for the ordinary discrete Fourier transform.

Result 3. The quadratic discrete Fourier transforms $x \leftrightarrow y$ and $x^{\prime} \leftrightarrow y^{\prime}$ associated withe same matrix matrix $F_{a}$, $a \in \mathbb{Z}_{d}$, satisfy the conservation rule

$$
\sum_{\alpha=0}^{d-1} \overline{y(\alpha)} y^{\prime}(\alpha)=\sum_{k=0}^{d-1} \overline{x(k)} x^{\prime}(k)
$$

where the common value is independent of a.

\subsection{Generalized Hadamard matrices}

The modulus of each matrix element of $F_{a}$ (with $a \in \mathbb{Z}_{d}$ ) is equal to $1 / \sqrt{d}$. Therefore, the unitary matrix $F_{a}$ turns out to be a generalized Hadamard matrix. We adopt here the following definition. A $d \times d$ generalized Hadamard matrix is a unitary matrix whose each entry has a modulus equal to $1 / \sqrt{d}[26]$. Note that the latter normalization, used in quantum information [27, 28], differs from the usual one according to which a $d \times d$ generalized Hadamard matrix $H$ is a complex matrix such that $H^{\dagger} H=d I_{d}$ and for which the modulus of each element is 1 [29]. In this respect, the generalized Hadamard matrix $H_{a}$ considered in [14] corresponds to $\sqrt{d} F_{a}$ up to permutations.

Example 1. By way of illustration, from (22) we get the familiar Hadamard matrices

$$
F_{0}=\frac{1}{\sqrt{2}}\left(\begin{array}{cc}
1 & -1 \\
1 & 1
\end{array}\right) \quad F_{1}=\frac{1}{\sqrt{2}}\left(\begin{array}{cc}
i & -i \\
1 & 1
\end{array}\right)
$$

for $d=2$ and

$$
F_{0}=\frac{1}{\sqrt{3}}\left(\begin{array}{ccc}
1 & \omega^{2} & \omega \\
1 & \omega & \omega^{2} \\
1 & 1 & 1
\end{array}\right) F_{1}=\frac{1}{\sqrt{3}}\left(\begin{array}{ccc}
\omega & 1 & \omega^{2} \\
\omega & \omega^{2} & 1 \\
1 & 1 & 1
\end{array}\right) F_{2}=\frac{1}{\sqrt{3}}\left(\begin{array}{ccc}
\omega^{2} & \omega & 1 \\
\omega^{2} & 1 & \omega \\
1 & 1 & 1
\end{array}\right)
$$


(with $\omega:=e^{i 2 \pi / 3}$ ) for $d=3$. Another example is

$$
F_{0}=\frac{1}{\sqrt{6}}\left(\begin{array}{cccccc}
1 & \tau & \tau^{2} & -1 & -\tau & -\tau^{2} \\
1 & \tau^{2} & -\tau & 1 & \tau^{2} & -\tau \\
1 & -1 & 1 & -1 & 1 & -1 \\
1 & -\tau & \tau^{2} & 1 & -\tau & \tau^{2} \\
1 & -\tau^{2} & -\tau & -1 & \tau^{2} & \tau \\
1 & 1 & 1 & 1 & 1 & 1
\end{array}\right)
$$

(with $\tau:=e^{-i \pi / 3}$ ) which readily follows from (22) for $d=6$ and $a=0$.

We sum up and complete this section with the following result (see also [14, 30]).

Result 4. The matrix

$$
F_{a}=\frac{1}{\sqrt{d}} \sum_{k=0}^{d-1} \sum_{\alpha=0}^{d-1} q^{(k+1)(d-k-1) a / 2-(k+1) \alpha} E_{k, \alpha}
$$

associated with the quadratic quantum Fourier transform (21) is a $d \times d$ generalized Hadamard matrix. It reduces the endomorphism associated with the operator $v_{0 a}$ :

$$
F_{a}^{\dagger} V_{0 a} F_{a}=q^{(d-1) a / 2} \sum_{\alpha=0}^{d-1} q^{-\alpha} E_{\alpha, \alpha}=q^{(d-1) a / 2}\left(\begin{array}{cccc}
1 & 0 & \ldots & 0 \\
0 & q^{-1} & \ldots & 0 \\
\vdots & \vdots & \ldots & \vdots \\
0 & 0 & \ldots & q^{-(d-1)}
\end{array}\right)
$$

where the matrix

$$
V_{0 a}:=\sum_{k=0}^{d-1} q^{k a} E_{k \ominus 1, k}=\left(\begin{array}{ccccc}
0 & q^{a} & 0 & \ldots & 0 \\
0 & 0 & q^{2 a} & \ldots & 0 \\
\vdots & \vdots & \vdots & \ldots & \vdots \\
0 & 0 & 0 & \ldots & q^{(d-1) a} \\
1 & 0 & 0 & \ldots & 0
\end{array}\right)
$$

represents the linear operator $v_{0 a}$ on the basis $B_{d}$.

\subsection{Generalized quadratic Gauss sums}

The Hadamard matrices $F_{a}$ and $F_{b}\left(a, b \in \mathbb{Z}_{d}\right)$ are connected to the inner product $\langle a \alpha \mid b \beta\rangle$. In fact, we have

$$
\langle a \alpha \mid b \beta\rangle=\left(F_{a}^{\dagger} F_{b}\right)_{\alpha \beta} .
$$

A direct calculation yields

$$
\langle a \alpha \mid b \beta\rangle=\frac{1}{d} \sum_{k=0}^{d-1} q^{k(d-k)(b-a) / 2-k(\beta-\alpha)}
$$


or

$$
\langle a \alpha \mid b \beta\rangle=\frac{1}{d} \sum_{k=0}^{d-1} e^{i \pi\left\{(a-b) k^{2}+[d(b-a)+2(\alpha-\beta)] k\right\} / d} .
$$

Hence, each matrix element of $F_{a}^{\dagger} F_{b}$ can be put in the form of a generalized quadratic Gauss sum $S(u, v, w)$ defined by [31]

$$
S(u, v, w):=\sum_{k=0}^{|w|-1} e^{i \pi\left(u k^{2}+v k\right) / w}
$$

where $u, v$ and $w$ are integers such that $u$ and $w$ are mutually prime, $u w \neq 0$ and $u w+v$ is even. In detail, we obtain

$$
\langle a \alpha \mid b \beta\rangle=\left(F_{a}^{\dagger} F_{b}\right)_{\alpha \beta}=\frac{1}{d} S(u, v, w)
$$

with the parameters

$$
u=a-b \quad v=-(a-b) d+2(\alpha-\beta) \quad w=d
$$

which ensure that $u w+v$ is necessarily even.

In the particular case $d=2$ (of special interest for qubits), we directly get

$$
\langle a \alpha \mid b \beta\rangle=\frac{1}{2}\left[1+e^{i \pi(b-a+2 \alpha-2 \beta) / 2}\right]
$$

which reduces to

$$
\langle a \alpha \mid a \beta\rangle=\delta_{\alpha, \beta} \quad b=a,
$$

and

$$
\langle a \alpha \mid b \beta\rangle=\frac{1}{2}(1 \pm i) \quad b \neq a,
$$

where the + sign corresponds to $b-a+2(\alpha-\beta)=1,-3$ and the - sign to $b-a+$ $2(\alpha-\beta)=-1,3$.

In the general case $d$ arbitrary (of interest for qudits), the calculation of $S(u, v, w)$ can be achieved by using the methods described in [31] (see also [32, 33, 34, 35]). The cases of interest for what follows are ( $u$ even, $v$ even, $w$ odd), ( $u$ odd, $v$ odd, $w$ odd) and ( $u$ odd, $v$ even, $w$ even). This leads to

Result 5. For $a \neq b, d$ arbitrary and $u+v+w$ odd, the inner product $\langle a \alpha \mid b \beta\rangle$ and the $\alpha \beta$-th element of the matrix $F_{a}^{\dagger} F_{b}$ follow from 
case $u=a-$ beven, $v=d(b-a)+2(\alpha-\beta)$ even, $w=d$ odd:

$$
\begin{gathered}
\langle a \alpha \mid b \beta\rangle=\left(F_{a}^{\dagger} F_{b}\right)_{\alpha \beta}=\sqrt{\frac{1}{w}}\left(\begin{array}{c}
u \\
w
\end{array}\right)_{L} \exp \left(-i \frac{\pi}{4}\left[w-1+\frac{u}{w}(u \backslash w)^{2} v^{2}\right]\right) \\
\text { case } u=a-b \text { odd, } v=d(b-a)+2(\alpha-\beta) \text { odd, } w=d \text { odd: } \\
\langle a \alpha \mid b \beta\rangle=\left(F_{a}^{\dagger} F_{b}\right)_{\alpha \beta}=\sqrt{\frac{1}{w}}\left(\begin{array}{c}
u \\
w
\end{array}\right)_{L} \exp \left(-i \frac{\pi}{4}\left[w-1+16 \frac{u}{w}(4 u \backslash w)^{2} v^{2}\right]\right) \\
\text { case } u=a-b \text { odd, } v=d(b-a)+2(\alpha-\beta) \text { even, } w=d \text { even: }
\end{gathered}
$$

so that the matrix $F_{a}^{\dagger} F_{b}$ is a Hadamard matrix for each case under consideration.

Finally, for $a=b$ and $d$ arbitrary we recover the orthonormality property (see (12))

$$
\langle a \alpha \mid a \beta\rangle=\delta_{\alpha, \beta}
$$

from a direct calculation of the right-hand side of (42).

\subsection{Mutually unbiased bases}

Speaking generally, two $d$-dimensional bases $B_{a}=\left\{|a \alpha\rangle: \alpha \in \mathbb{Z}_{d}\right\}$ and $B_{b}=\{|b \beta\rangle$ : $\left.\beta \in \mathbb{Z}_{d}\right\}$ are said to be mutually unbiased if and only if

$$
|\langle a \alpha \mid b \beta\rangle|=\delta_{a, b} \delta_{\alpha, \beta}+\left(1-\delta_{a, b}\right) \frac{1}{\sqrt{d}}
$$

for any $\alpha$ and $\beta$ in the ring $\mathbb{Z}_{d}$. It is well-known that the number of mutually unbiased bases (MUBs) in the Hilbert space $\mathbb{C}^{d}$ cannot be greater than $d+1$ [36, 37, 38, 39]. In fact, the maximum number $d+1$ is attained when $d$ is the power of a prime number [38, 39]. Despite a considerable amount of works, the maximum number of MUBs is unknown when $d$ is not a power of a prime. In this respect, several numerical studies strongly suggest that there are only three MUBs for $d=6$ (see for example [27, 28, 40, 41, 42]). MUBs are closely connected with the concept of complementarity in quantum mechanics. There are of paramount importance in classical information theory (Kerdock codes and network communication protocols) [39, 43], in quantum information theory (quantum cryptography and quantum state tomography) [44] and in the solution of the Mean King problem [45, 46, 47, 48, 49, 50]. Recently, it was pointed out and confirmed 
that MUBs are also of central importance in the formalism of Feynman path integrals [51, 52]. Finally, it should be emphasized that the concept of MUBs also exists in infinite dimension [53]. There are numerous ways of constructing sets of MUBs. Most of them are based on discrete Fourier analysis over Galois fields and Galois rings, discrete Wigner functions, generalized Pauli matrices, mutually orthogonal Latin squares, finite geometry methods and Lie-like approaches (see Refs. [14, 25, 27, 28, 30, 36, 37, 38, 39, 40, 41, 42, 43, 44, 45, 46, 47, 48, 49, 50, 54, 55, 56, 57, 58, 59, 60, 61, 62, 63, 64] for an nonexhaustive list of references).

\subsubsection{Case $d$ prime}

We have the following important result. (See also [64] for a recent alternative grouptheoretical approach to the case $d$ prime.)

Result 6. In the case where $d=p$ is a prime number (even or odd), one has

$$
|\langle a \alpha \mid b \beta\rangle|=\left|\left(F_{a}^{\dagger} F_{b}\right)_{\alpha \beta}\right|=\frac{1}{\sqrt{p}} \quad a \neq b
$$

for $a, b, \alpha, \beta \in \mathbb{Z}_{p}$. Therefore, the $p+1$ bases $B_{0}, B_{1}, \ldots, B_{p}$ constitute a complete set of MUBs in $\mathbb{C}^{p}$.

The proof easily follows from the calculation of the modulus of $S(a-b, p b-p a+$ $2 \alpha-2 \beta, p)$ from (46), (49), (50) and (51). As a consequence, the bases $B_{a}$, with $a=$ $0,1, \ldots, p-1$, are $p$ MUBs in the sense that they satisfy (53) for any $a, b, \alpha$ and $\beta$ in the Galois field $\mathbb{F}_{p}$. Obviously, each of the bases $B_{a}$ (with $a=0,1, \ldots, p-1$ ) is mutually unbiased with the computational basis $B_{p}$. This completes the proof. Note that Result 6 can be proved as well from the developments in [14].

As two typical examples, let us examine the cases $d=2$ and 3.

Example 2: case $d=2$. In this case, relevant for a spin $j=1 / 2$ or for a qubit, we have $q=-1$ and $a, \alpha \in \mathbb{Z}_{2}$. The matrices of the operators $v_{0 a}$ are

$$
V_{00}=\left(\begin{array}{ll}
0 & 1 \\
1 & 0
\end{array}\right) \quad V_{01}=\left(\begin{array}{cc}
0 & -1 \\
1 & 0
\end{array}\right)
$$

By using the notation

$$
\alpha:=\left|\frac{1}{2}, \frac{1}{2}\right\rangle \quad \beta:=\left|\frac{1}{2},-\frac{1}{2}\right\rangle
$$


familiar in quantum chemistry ( $\alpha$ is a spinorbital for spin up and $\beta$ for spin down), the $d+1=3$ MUBs are

$$
\begin{array}{lll}
B_{0}:|00\rangle=\frac{1}{\sqrt{2}}(\alpha+\beta) & |01\rangle=-\frac{1}{\sqrt{2}}(\alpha-\beta) \\
B_{1}:|10\rangle=i \frac{1}{\sqrt{2}}(\alpha-i \beta) & |11\rangle=-i \frac{1}{\sqrt{2}}(\alpha+i \beta) \\
B_{2}:|0\rangle=\alpha \quad|1\rangle=\beta . &
\end{array}
$$

Example 3: case $d=3$. This case corresponds to a spin $j=1$ or to a qutrit. Here, we have $q=\exp (i 2 \pi / 3)$ and $a, \alpha \in \mathbb{Z}_{3}$. The matrices of the operators $v_{0 a}$ are

$$
V_{00}=\left(\begin{array}{ccc}
0 & 1 & 0 \\
0 & 0 & 1 \\
1 & 0 & 0
\end{array}\right) \quad V_{01}=\left(\begin{array}{ccc}
0 & q & 0 \\
0 & 0 & q^{2} \\
1 & 0 & 0
\end{array}\right) \quad V_{02}=\left(\begin{array}{ccc}
0 & q^{2} & 0 \\
0 & 0 & q \\
1 & 0 & 0
\end{array}\right)
$$

The $d+1=4$ MUBs read

$$
\begin{aligned}
B_{0}: \quad|00\rangle & =\frac{1}{\sqrt{3}}(|0\rangle+|1\rangle+|2\rangle) \\
|01\rangle & =\frac{1}{\sqrt{3}}\left(q^{2}|0\rangle+q|1\rangle+|2\rangle\right) \\
|02\rangle & =\frac{1}{\sqrt{3}}\left(q|0\rangle+q^{2}|1\rangle+|2\rangle\right) \\
B_{1}: \quad|10\rangle & =\frac{1}{\sqrt{3}}(q|0\rangle+q|1\rangle+|2\rangle) \\
|11\rangle & =\frac{1}{\sqrt{3}}\left(|0\rangle+q^{2}|1\rangle+|2\rangle\right) \\
|12\rangle & =\frac{1}{\sqrt{3}}\left(q^{2}|0\rangle+|1\rangle+|2\rangle\right) \\
B_{2}: \quad|20\rangle & =\frac{1}{\sqrt{3}}\left(q^{2}|0\rangle+q^{2}|1\rangle+|2\rangle\right) \\
|21\rangle & =\frac{1}{\sqrt{3}}(q|0\rangle+|1\rangle+|2\rangle) \\
|22\rangle & =\frac{1}{\sqrt{3}}(|0\rangle+q|1\rangle+|2\rangle) \\
B_{3}: \quad|0\rangle & =|1,1\rangle \quad|1\rangle=|1,0\rangle \quad|2\rangle=|1,-1\rangle .
\end{aligned}
$$

It should be observed that $B_{0}$ (respectively, $B_{1}$ and $B_{2}$ ) can be associated with the vector (respectively, projective) irreducible representations of the group $C_{3}$. 


\subsubsection{Case $d$ power of a prime}

Different constructions of MUBs in the case where $d$ is a power of a prime were achieved by numerous authors from algebraical and geometrical techniques (see for instance [38, 39, 54, 55, 56, 57, 58, 59, 60, 61, 62, 63] and references therein). We want to show here, through an example for $d=4$, how our angular momentum approach can be useful for addressing this case.

Example 4: case $d=4$. This case corresponds to a spin $j=3 / 2$. Here, we have $q=i$ and $a, \alpha \in \mathbb{Z}_{4}$. Equations (20) and (28) can be applied to this case too. However, the resulting bases $B_{0}, B_{1}, B_{2}, B_{3}$ and $B_{4}$ do not constitute a complete system of MUBs ( $d=4$ is not a prime number). Nevertheless, it is possible to find $d+1=5 \mathrm{MUBs}$ because $d=2^{2}$ is the power of a prime number. This can be achieved by replacing the space $\mathcal{E}(4)$ spanned by $\{|3 / 2, m\rangle: m=3 / 2,1 / 2,-1 / 2,-3 / 2\}$ by the tensor product space $\mathcal{E}(2) \otimes \mathcal{E}(2)$ spanned by the basis

$$
\{\alpha \otimes \alpha, \alpha \otimes \beta, \beta \otimes \alpha, \beta \otimes \beta\}
$$

The space $\mathcal{E}(2) \otimes \mathcal{E}(2)$ is associated with the coupling of two spin angular momenta $j_{1}=1 / 2$ and $j_{2}=1 / 2$ or two qubits (in the vector $u \otimes v, u$ and $v$ correspond to $j_{1}$ and $j_{2}$, respectively).

In addition to the basis (65), it is possible to find other bases of $\mathcal{E}(2) \otimes \mathcal{E}(2)$ which are mutually unbiased. The $d=4$ MUBs besides the canonical or computational basis (65) can be constructed from the eigenvectors

$$
|a b \alpha \beta\rangle:=|a \alpha\rangle \otimes|b \beta\rangle
$$

of the operators

$$
w_{a b}:=v_{0 a} \otimes v_{0 b}
$$

(the vectors $|a \alpha\rangle$ and $|b \beta\rangle$ refer to the two spaces $\mathcal{E}(2)$ ). As a result, we have the $d+1=5$ following MUBs where $\lambda=(1-i) / 2$ and $\mu=i \lambda$.

The canonical basis:

$$
\alpha \otimes \alpha \quad \alpha \otimes \beta \quad \beta \otimes \alpha \quad \beta \otimes \beta
$$

The $w_{00}$ basis:

$$
|0000\rangle=\frac{1}{2}(\alpha \otimes \alpha+\alpha \otimes \beta+\beta \otimes \alpha+\beta \otimes \beta)
$$




$$
\begin{aligned}
|0001\rangle & =\frac{1}{2}(\alpha \otimes \alpha-\alpha \otimes \beta+\beta \otimes \alpha-\beta \otimes \beta) \\
|0010\rangle & =\frac{1}{2}(\alpha \otimes \alpha+\alpha \otimes \beta-\beta \otimes \alpha-\beta \otimes \beta) \\
|0011\rangle & =\frac{1}{2}(\alpha \otimes \alpha-\alpha \otimes \beta-\beta \otimes \alpha+\beta \otimes \beta) .
\end{aligned}
$$

The $w_{11}$ basis:

$$
\begin{aligned}
|1100\rangle & =\frac{1}{2}(\alpha \otimes \alpha+i \alpha \otimes \beta+i \beta \otimes \alpha-\beta \otimes \beta) \\
|1101\rangle & =\frac{1}{2}(\alpha \otimes \alpha-i \alpha \otimes \beta+i \beta \otimes \alpha+\beta \otimes \beta) \\
|1110\rangle & =\frac{1}{2}(\alpha \otimes \alpha+i \alpha \otimes \beta-i \beta \otimes \alpha+\beta \otimes \beta) \\
|1111\rangle & =\frac{1}{2}(\alpha \otimes \alpha-i \alpha \otimes \beta-i \beta \otimes \alpha-\beta \otimes \beta) .
\end{aligned}
$$

The $w_{01}$ basis:

$$
\begin{aligned}
& \lambda|0100\rangle+\mu|0111\rangle=\frac{1}{2}(\alpha \otimes \alpha+\alpha \otimes \beta-i \beta \otimes \alpha+i \beta \otimes \beta) \\
& \mu|0100\rangle+\lambda|0111\rangle=\frac{1}{2}(\alpha \otimes \alpha-\alpha \otimes \beta+i \beta \otimes \alpha+i \beta \otimes \beta) \\
& \lambda|0101\rangle+\mu|0110\rangle=\frac{1}{2}(\alpha \otimes \alpha-\alpha \otimes \beta-i \beta \otimes \alpha-i \beta \otimes \beta) \\
& \mu|0101\rangle+\lambda|0110\rangle=\frac{1}{2}(\alpha \otimes \alpha+\alpha \otimes \beta+i \beta \otimes \alpha-i \beta \otimes \beta) .
\end{aligned}
$$

The $w_{10}$ basis:

$$
\begin{aligned}
\lambda|1000\rangle+\mu|1011\rangle & =\frac{1}{2}(\alpha \otimes \alpha-i \alpha \otimes \beta+\beta \otimes \alpha+i \beta \otimes \beta) \\
\mu|1000\rangle+\lambda|1011\rangle & =\frac{1}{2}(\alpha \otimes \alpha+i \alpha \otimes \beta-\beta \otimes \alpha+i \beta \otimes \beta) \\
\lambda|1001\rangle+\mu|1010\rangle & =\frac{1}{2}(\alpha \otimes \alpha+i \alpha \otimes \beta+\beta \otimes \alpha-i \beta \otimes \beta) \\
\mu|1001\rangle+\lambda|1010\rangle & =\frac{1}{2}(\alpha \otimes \alpha-i \alpha \otimes \beta-\beta \otimes \alpha-i \beta \otimes \beta) .
\end{aligned}
$$

It is to be noted that the vectors of the $w_{00}$ and $w_{11}$ bases are not intricated (i.e., each vector is the direct product of two vectors) while the vectors of the $w_{01}$ and $w_{10}$ bases are intricated (i.e., each vector is not the direct product of two vectors). To be more precise, the degree of intrication of the state vectors for the bases $w_{00}, w_{11}, w_{01}$ and $w_{10}$ can be determined in the following way. In arbitrary dimension $d$, let

$$
|\Phi\rangle=\sum_{k=0}^{d-1} \sum_{l=0}^{d-1} a_{k l}|k\rangle \otimes|l\rangle
$$


be a double qudit state vector. Then, it can be shown that the determinant of the $d \times d$ matrix $A=\left(a_{k l}\right)$ satisfies

$$
0 \leq|\operatorname{det} A| \leq \frac{1}{\sqrt{d^{d}}}
$$

as proved in the Albouy thesis [65, 66]. The case $\operatorname{det} A=0$ corresponds to the absence of intrication while the case

$$
|\operatorname{det} A|=\frac{1}{\sqrt{d^{d}}}
$$

corresponds to a maximal intrication. As an illustration, we obtain that all the state vectors for $w_{00}$ and $w_{11}$ are not intricated and that all the state vectors for $w_{01}$ and $w_{10}$ are maximally intricated.

\subsubsection{Case $d$ arbitrary}

In the special case where $u=1$, the generalized Gauss sum $S(1,-d+2 \alpha-2 \beta, d)$ can be easily calculated for $d$ arbitrary by means of the reciprocity theorem [31]

$$
S(u, v, w)=\sqrt{\left|\frac{w}{u}\right|} e^{i \pi\left[\operatorname{sgn}(u w)-v^{2} /(u w)\right] / 4} S(-w,-v, u) .
$$

This leads to the following particular result.

Result 7. For $d$ arbitrary and $b=a \ominus 1$, one has

$$
\left\langle a \alpha \mid a_{-1} \beta\right\rangle=\frac{1}{\sqrt{d}} e^{i \pi\left[1-(d-2 \alpha+2 \beta)^{2} / d\right] / 4} \Rightarrow\left|\left\langle a \alpha \mid a_{-1} \beta\right\rangle\right|=\frac{1}{\sqrt{d}} \quad a_{-1}=a \ominus 1 .
$$

Therefore, the three bases $B_{a \ominus 1}, B_{a}$ and $B_{d}$ are mutually unbiased in $\mathbb{C}^{d}$.

This result is in agreement with a well-known result proved in many papers from quite distinct ways (see for instance [41]). We thus recover, from an approach based on generalized Gauss sums, that for $d$ arbitrary the minimum number of MUBs is 3 .

Another special case, viz., $u=2(\Rightarrow d \geq 3)$, is worth of value. The application of the reciprocity theorem gives here

Result 8. For $d \geq 3$ and $b=a \ominus 2$, one has

$$
\begin{aligned}
\left\langle a \alpha \mid a_{-2} \beta\right\rangle & =\frac{1}{\sqrt{d}} \frac{1}{\sqrt{2}} e^{i \pi\left[1-2(\alpha-\beta)^{2} / d\right] / 4}\left[1+e^{i \pi(-d+2 \alpha-2 \beta) / 2}\right] \\
& \Rightarrow\left|\left\langle a \alpha \mid a_{-2} \beta\right\rangle\right|=\sqrt{\frac{2}{d}}\left|\cos \left[\frac{\pi}{4}(d-2 \alpha+2 \beta)\right]\right| \quad a_{-2}=a \ominus 2 .(90)
\end{aligned}
$$


Therefore, the bases $B_{a \ominus 2}$ and $B_{a}$ cannot be mutually unbiased in $\mathbb{C}^{d}$ for $d$ even with $d \geq 4$. In marked contrast, the bases $B_{a \ominus 2}$ and $B_{a}$ are unbiased for $d$ odd with $d \geq 3$ ( $d$ prime or not prime).

Going back to the Hadamard matrices, let us remark that, for $d$ arbitrary, if $B_{a}$ and $B_{b}$ are two MUBs associated with the Hadamard matrices $F_{a}$ and $F_{b}$ (respectively), then $F_{a}^{\dagger} F_{b}$ is a Hadamard matrix too. However, for $d$ arbitrary, if $F_{a}$ and $F_{b}$ are two Hadamard matrices associated with the bases $B_{a}$ and $B_{b}$ (respectively), the product $F_{a}^{\dagger} F_{b}$ is not in general a Hadamard matrix.

\section{Unitary group and generalized Pauli group}

\subsection{Weyl pairs}

We continue with the general case where $d$ is arbitrary. The operator $v_{0 a}$ can be expressed as

$$
v_{0 a}=\sum_{k=0}^{d-1} q^{k a}|k \ominus 1\rangle\left\langle k\left|\Leftrightarrow v_{0 a}=\sum_{m=-j}^{j} q^{(j-m) a}\right| j, m \oplus 1\right\rangle\langle j, m|
$$

so that

$$
v_{0 a}|k\rangle=q^{k a}|k \ominus 1\rangle \Leftrightarrow v_{0 a}|j, m\rangle=q^{(j-m) a}|j, m \oplus 1\rangle
$$

where $q=\exp (2 \pi i / d)$. The operators $x$ (the flip or shift operator) and $z$ (the clock operator), used in quantum information and quantum computation (see for instance [67, 68]), can be derived from the generic operator $v_{0 a}$ as follows

$$
x:=v_{00} \quad z:=\left(v_{00}\right)^{\dagger} v_{01} .
$$

Therefore, we get

$$
x=\sum_{k=0}^{d-1}|k \ominus 1\rangle\langle k|=| d-1\rangle\langle 0|+| 0\rangle\langle 1|+\ldots+| d-2\rangle\langle d-1|
$$

and

$$
z=\sum_{k=0}^{d-1} q^{k}|k\rangle\langle k|=| 0\rangle\langle 0|+q| 1\rangle\left\langle 1\left|+\ldots+q^{d-1}\right| d-1\right\rangle\langle d-1| .
$$

The action of $x$ and $z$ on the basis $B_{d}$ of $\mathcal{E}(d)$ is given by the ladder relation

$$
x|k\rangle=|k \ominus 1\rangle \Leftrightarrow x|j, m\rangle=\left(1-\delta_{m, j}\right)|j, m+1\rangle+\delta_{m, j}|j,-j\rangle
$$


and the phase relation

$$
z|k\rangle=q^{k}|k\rangle \Leftrightarrow z|j, m\rangle=q^{j-m}|j, m\rangle
$$

Alternatively, the action of $x$ and $z$ on any basis $B_{a}(a=0,1, \ldots, d-1)$ of $\mathcal{E}(d)$ reads

$$
x|a \alpha\rangle=q^{(d-1) a / 2-\alpha}\left|a \alpha_{a}\right\rangle \quad \alpha_{a}=\alpha \oplus a \quad \Rightarrow \quad x|0 \alpha\rangle=q^{-\alpha}|0 \alpha\rangle
$$

and

$$
z|a \alpha\rangle=q^{-1}\left|a \alpha_{-1}\right\rangle \quad \alpha_{-1}=\alpha \ominus 1
$$

Equations (96) and (97), on one side, and Eqs. (98) and (99), on the other side, show that the flip or clock character for $x$ and $z$ is basis-dependent. The relationship between $x$ and $z$ can be understood via the following

Result 9. The unitary operators $x$ and $z$ are cyclic and q-commute:

$$
x^{d}=z^{d}=I \quad x z-q z x=0 .
$$

They are connected by

$$
x=f^{\dagger} z f \Leftrightarrow z=f x f^{\dagger}
$$

where the Fourier operator

$$
f:=\frac{1}{\sqrt{d}} \sum_{k=0}^{d-1} \sum_{k^{\prime}=0}^{d-1} q^{-k k^{\prime}}|k\rangle\left\langle k^{\prime}\right|
$$

is unitary and satisfies

$$
f^{4}=1
$$

The operators $x$ and $z$ are isospectral operators with the common spectrum $\left\{1, q, \ldots, q^{d-1}\right\}$.

A direct proof of Result 9 can be obtained by switching to the matrices

$$
X=\sum_{k=0}^{d-1} E_{k \ominus 1, k}=\left(\begin{array}{ccccc}
0 & 1 & 0 & \ldots & 0 \\
0 & 0 & 1 & \ldots & 0 \\
\vdots & \vdots & \vdots & \ldots & \vdots \\
0 & 0 & 0 & \ldots & 1 \\
1 & 0 & 0 & \ldots & 0
\end{array}\right)
$$




$$
Z=\sum_{k=0}^{d-1} q^{k} E_{k, k}=\left(\begin{array}{ccccc}
1 & 0 & 0 & \ldots & 0 \\
0 & q & 0 & \ldots & 0 \\
0 & 0 & q^{2} & \ldots & 0 \\
\vdots & \vdots & \vdots & \ldots & \vdots \\
0 & 0 & 0 & \ldots & q^{d-1}
\end{array}\right)
$$

of the operators $x$ and $z$, in the basis $B_{d}$ (cf. (97) and (98)). Let $F$ be the matrix of the linear operator $f$ in the basis $B_{d}$. The reduction by means of $F$ of the endomorphism associated with the matrix $X$ yields the matrix $Z$. In other words, the diagonalization of $X$ can be achieved with the help of the matrix $F$ via $Z=F X F^{\dagger}$. Note that the matrix $F$ is connected to $F_{0}$ by

$$
F=\left(F_{0} S\right)^{\dagger} \quad S:=\sum_{\beta=0}^{d-1} q^{\beta} E_{\beta, d-\beta}
$$

where $S$ acts as a pseudopermutation.

In view of (100), the pair $(x, z)$ is called a Weyl pair. Weyl pairs were originally introduced in finite quantum mechanics [69] and used for the construction of unitary bases in finite-dimensional Hilbert spaces [70]. It should be noted that matrices of type $X$ and $Z$ were introduced long time ago by Sylvester [71] in order to solve the matrix equation $P X=X Q$; in addition, such matrices were used by Morris [72] to define generalized Clifford algebras in connection with quaternion algebras and division rings. Besides the Weyl pair $(x, z)$, other pairs can be formed with the operators $v_{0 a}$ and $z$. Indeed, any operator $v_{0 a}\left(a \in \mathbb{Z}_{d}\right)$ can be generated from $x$ and $z$ since

$$
v_{0 a}=x z^{a}
$$

Thus, Eq. (100) can be generalized as

$$
e^{-i \pi(d-1) a}\left(v_{0 a}\right)^{d}=z^{d}=I \quad v_{0 a} z-q z v_{0 a}=0 .
$$

Therefore, the pair $\left(v_{0 a}, z\right)$ is a Weyl pair for $(d-1) a$ even.

\subsection{Generalized Pauli matrices}

For $d=2$ the $q$-commutation relation of $x$ and $z$ reduces to an anticommutation relation. In fact, Eq. (100) with $d=2$ can be particularized to the relations

$$
x^{2}=z^{2}=I \quad x z+z x=0
$$

which are reminiscent of relations satisfied by the Pauli matrices. Hence, we understand that the matrices $X$ and $Z$ for $d$ arbitrary can be used as an integrity basis for producing 
generalized Pauli matrices $[37,38,39,54,55,57,58,59,60,73,74,75,76,77,78,79$, 80, 81, 82]. Let us develop this point.

For $d$ arbitrary, we define the operators

$$
u_{a b}=x^{a} z^{b} \quad a, b \in \mathbb{Z}_{d}
$$

The operators $u_{a b}$ shall be referred as generalized Pauli operators and their matrices as generalized Pauli matrices. They satisfy the ladder-phase relation

$$
u_{a b}|k\rangle=q^{k b}|k \ominus a\rangle \Leftrightarrow u_{a b}|j, m\rangle=q^{(j-m) b}|j, m \oplus a\rangle
$$

from which we can derive the following result.

Result 10. The $d^{2}$ operators $u_{a b}$, with $a, b \in \mathbb{Z}_{d}$, are unitary and obey the multiplication rule

$$
u_{a b} u_{a^{\prime} b^{\prime}}=q^{-b a^{\prime}} u_{a^{\prime \prime} b^{\prime \prime}} \quad a^{\prime \prime}:=a \oplus a^{\prime} \quad b^{\prime \prime}:=b \oplus b^{\prime} .
$$

Therefore, the commutator and the anticommutator of $u_{a b}$ and $u_{a^{\prime} b^{\prime}}$ are given by

$$
\left[u_{a b}, u_{a^{\prime} b^{\prime}}\right]_{ \pm}=\left(q^{-b a^{\prime}} \pm q^{-a b^{\prime}}\right) u_{a^{\prime \prime} b^{\prime \prime}} \quad a^{\prime \prime}:=a \oplus a^{\prime} \quad b^{\prime \prime}:=b \oplus b^{\prime}
$$

Furthermore, they are orthogonal with respect to the Hilbert-Schmidt inner product

$$
\operatorname{Tr}_{\mathcal{E}(d)}\left[\left(u_{a b}\right)^{\dagger} u_{a^{\prime} b^{\prime}}\right]=d \delta_{a, a^{\prime}} \delta_{b, b^{\prime}}
$$

where the trace is taken on the d-dimensional space $\mathcal{E}(d)$.

As a corollary of Result 10, we have

$$
\left[u_{a b}, u_{a^{\prime} b^{\prime}}\right]_{-}=0 \Leftrightarrow a b^{\prime} \ominus b a^{\prime}=0
$$

and

$$
\left[u_{a b}, u_{a^{\prime} b^{\prime}}\right]_{+}=0 \Leftrightarrow a b^{\prime} \ominus b a^{\prime}=\frac{1}{2} d
$$

This yields two consequences. First, Eq. (116) shows that all anticommutators $\left[u_{a b}, u_{a^{\prime} b^{\prime}}\right]_{+}$ are different from 0 if $d$ is an odd integer. Second, from Eq. (115) we have the important result that, for $d$ arbitrary, each of the three disjoint sets

$$
\begin{aligned}
& e_{0 \bullet}:=\left\{u_{0 a}=z^{a}: a=1,2, \ldots, d-1\right\} \\
& e_{\bullet \bullet}:=\left\{u_{a a}=x^{a} z^{a}: a=1,2, \ldots, d-1\right\} \\
& e_{\bullet}:=\left\{u_{a 0}=x^{a}: a=1,2, \ldots, d-1\right\}
\end{aligned}
$$


consist of $d-1$ mutually commuting operators. The three sets $e_{0 \bullet}, e_{\bullet \bullet}$ and $e_{\bullet 0}$ are associated with three MUBs. This is in agreement with the fact that the bases $B_{0}, B_{1}$ and $B_{d}$ are three MUBs for $d$ arbitrary $\left(v_{00}=x \in e_{\bullet 0}, v_{01}=x z \in e_{\bullet \bullet}\right.$ and $z \in e_{0 \bullet}$ are associated with $B_{0}, B_{1}$ and $B_{d}$, respectively).

By way of illustration, let us give the matrices in the basis $B_{d}$ of the operators $u_{a b}$ for $d=2,3$ and 4 .

Example 5: case $d=2$. For $d=2 \Leftrightarrow j=1 / 2(\Rightarrow q=-1)$, the matrices in the two sets

$$
\begin{aligned}
& E_{0}:=\left\{I_{2}=X^{0} Z^{0}, X=X^{1} Z^{0} \equiv V_{00}\right\} \\
& E_{1}:=\left\{Z=X^{0} Z^{1}, Y=X^{1} Z^{1} \equiv V_{01}\right\}
\end{aligned}
$$

corresponding to the four operators $u_{a b}$ are

$$
I_{2}=\left(\begin{array}{ll}
1 & 0 \\
0 & 1
\end{array}\right) \quad X=\left(\begin{array}{ll}
0 & 1 \\
1 & 0
\end{array}\right) \quad Z=\left(\begin{array}{cc}
1 & 0 \\
0 & -1
\end{array}\right) \quad Y=\left(\begin{array}{cc}
0 & -1 \\
1 & 0
\end{array}\right)
$$

In terms of the usual (Hermitian and unitary) Pauli matrices $\sigma_{x}, \sigma_{y}$ and $\sigma_{z}$, we have

$$
X=\sigma_{x} \quad Y=-i \sigma_{y} \quad Z=\sigma_{z} .
$$

The matrices $X, Y$ and $Z$ are thus identical to the Pauli matrices up to a phase factor for $Y$. This phase factor is the price one has to pay in order to get a systematic generalization of Pauli matrices in arbitrary dimension.

Example 6: case $d=3$. For $d=3 \Leftrightarrow j=1(\Rightarrow q=\exp (i 2 \pi / 3))$, the matrices in the three sets

$$
\begin{aligned}
& E_{0}:=\left\{X^{0} Z^{0}, X^{1} Z^{0} \equiv V_{00}, X^{2} Z^{0}\right\} \\
& E_{1}:=\left\{X^{0} Z^{1}, X^{1} Z^{1} \equiv V_{01}, X^{2} Z^{1}\right\} \\
& E_{2}:=\left\{X^{0} Z^{2}, X^{1} Z^{2} \equiv V_{02}, X^{2} Z^{2}\right\}
\end{aligned}
$$

corresponding to the nine operators $u_{a b}$ are

$$
\begin{aligned}
& I_{3}=\left(\begin{array}{lll}
1 & 0 & 0 \\
0 & 1 & 0 \\
0 & 0 & 1
\end{array}\right) \quad X=\left(\begin{array}{lll}
0 & 1 & 0 \\
0 & 0 & 1 \\
1 & 0 & 0
\end{array}\right) \quad X^{2}=\left(\begin{array}{lll}
0 & 0 & 1 \\
1 & 0 & 0 \\
0 & 1 & 0
\end{array}\right) \\
& Z=\left(\begin{array}{ccc}
1 & 0 & 0 \\
0 & q & 0 \\
0 & 0 & q^{2}
\end{array}\right) \quad X Z=\left(\begin{array}{ccc}
0 & q & 0 \\
0 & 0 & q^{2} \\
1 & 0 & 0
\end{array}\right) \quad X^{2} Z=\left(\begin{array}{ccc}
0 & 0 & q^{2} \\
1 & 0 & 0 \\
0 & q & 0
\end{array}\right)
\end{aligned}
$$




$$
Z^{2}=\left(\begin{array}{ccc}
1 & 0 & 0 \\
0 & q^{2} & 0 \\
0 & 0 & q
\end{array}\right) \quad X Z^{2}=\left(\begin{array}{ccc}
0 & q^{2} & 0 \\
0 & 0 & q \\
1 & 0 & 0
\end{array}\right) \quad X^{2} Z^{2}=\left(\begin{array}{ccc}
0 & 0 & q \\
1 & 0 & 0 \\
0 & q^{2} & 0
\end{array}\right)
$$

These generalized Pauli matrices differ from the Gell-Mann matrices and Okubo matrices used for $S U(3)$ in particle physics with three flavors of quarks [83, 84, 85]. They constitute a natural extension based on Weyl pairs of the Pauli matrices in dimension $d=3$.

Example 7: case $d=4$. For $d=4 \Leftrightarrow j=3 / 2(\Rightarrow q=i)$, the matrices in the four sets

$$
\begin{aligned}
& E_{0}:=\left\{X^{0} Z^{0}, X^{1} Z^{0} \equiv V_{00}, X^{2} Z^{0}, X^{3} Z^{0}\right\} \\
& E_{1}:=\left\{X^{0} Z^{1}, X^{1} Z^{1} \equiv V_{01}, X^{2} Z^{1}, X^{3} Z^{1}\right\} \\
& E_{2}:=\left\{X^{0} Z^{2}, X^{1} Z^{2} \equiv V_{02}, X^{2} Z^{2}, X^{3} Z^{2}\right\} \\
& E_{3}:=\left\{X^{0} Z^{3}, X^{1} Z^{3} \equiv V_{03}, X^{2} Z^{3}, X^{3} Z^{3}\right\}
\end{aligned}
$$

corresponding to the 16 operators $u_{a b}$ are

$$
\begin{array}{ccc}
I_{4}=\left(\begin{array}{cccc}
1 & 0 & 0 & 0 \\
0 & 1 & 0 & 0 \\
0 & 0 & 1 & 0 \\
0 & 0 & 0 & 1
\end{array}\right) & X=\left(\begin{array}{cccc}
0 & 1 & 0 & 0 \\
0 & 0 & 1 & 0 \\
0 & 0 & 0 & 1 \\
1 & 0 & 0 & 0
\end{array}\right) \\
X^{2}=\left(\begin{array}{cccc}
0 & 0 & 1 & 0 \\
0 & 0 & 0 & 1 \\
1 & 0 & 0 & 0 \\
0 & 1 & 0 & 0
\end{array}\right) & X^{3}=\left(\begin{array}{cccc}
0 & 0 & 0 & 1 \\
1 & 0 & 0 & 0 \\
0 & 1 & 0 & 0 \\
0 & 0 & 1 & 0
\end{array}\right) \\
Z= & \left(\begin{array}{cccc}
1 & 0 & 0 & 0 \\
0 & i & 0 & 0 \\
0 & 0 & -1 & 0 \\
1 & 0 & 0 & -i
\end{array}\right) & X Z=\left(\begin{array}{cccc}
0 & i & 0 & 0 \\
0 & 0 & -1 & 0 \\
0 & 0 & 0 & -i \\
1 & 0 & 0 & 0
\end{array}\right) \\
X^{2} Z=\left(\begin{array}{cccc}
0 & 0 & -1 & 0 \\
0 & 0 & 0 & -i \\
1 & 0 & 0 & 0 \\
0 & i & 1 & 0
\end{array}\right) & X^{3} Z=\left(\begin{array}{cccc}
0 & 0 & 0 & -i \\
1 & 0 & 0 & 0 \\
0 & i & 0 & 0 \\
0 & 0 & -1 & 0
\end{array}\right) \\
Z^{2}= & \left(\begin{array}{cccc}
1 & 0 & 0 & 0 \\
0 & -1 & 0 & 0 \\
0 & 0 & 1 & 0 \\
0 & 0 & 0 & -1
\end{array}\right) & X Z^{2}=\left(\begin{array}{cccc}
0 & -1 & 0 & 0 \\
0 & 0 & 1 & 0 \\
0 & 0 & 0 & -1 \\
1 & 0 & 0 & 0
\end{array}\right)
\end{array}
$$




$$
\begin{array}{rlrl}
X^{2} Z^{2} & =\left(\begin{array}{cccc}
0 & 0 & 1 & 0 \\
0 & 0 & 0 & -1 \\
1 & 0 & 0 & 0 \\
0 & -1 & 0 & 0
\end{array}\right) & X^{3} Z^{2}=\left(\begin{array}{cccc}
0 & 0 & 0 & -1 \\
1 & 0 & 0 & 0 \\
0 & -1 & 0 & 0 \\
0 & 0 & 1 & 0
\end{array}\right) \\
Z^{3}=\left(\begin{array}{cccc}
1 & 0 & 0 & 0 \\
0 & -i & 0 & 0 \\
0 & 0 & -1 & 0 \\
0 & 0 & 0 & i
\end{array}\right) & X Z^{3}=\left(\begin{array}{cccc}
0 & -i & 0 & 0 \\
0 & 0 & -1 & 0 \\
0 & 0 & 0 & i \\
1 & 0 & 0 & 0
\end{array}\right) \\
X^{2} Z^{3}=\left(\begin{array}{cccc}
0 & 0 & -1 & 0 \\
0 & 0 & 0 & i \\
1 & 0 & 0 & 0 \\
0 & -i & 0 & 0
\end{array}\right) & X^{3} Z^{3}=\left(\begin{array}{cccc}
0 & 0 & 0 & i \\
1 & 0 & 0 & 0 \\
0 & -i & 0 & 0 \\
0 & 0 & -1 & 0
\end{array}\right) .
\end{array}
$$

These generalized Pauli matrices are linear combinations of the generators of the chain $S U(4) \supset S U(3) \supset S U(2)$ in particle physics with four flavors of quarks [86, 87, 88].

For $d$ arbitrary, the generalized Pauli matrices arising from (110) are different from the generalized Gell-Mann $\lambda$ matrices introduced in [89]. The generalized $\lambda$ matrices are Hermitian and adapted to the chain of groups $S U(d) \supset S U(d-1) \supset \ldots \supset S U(2)$ while the matrices $X^{a} Z^{b}$ are unitary and closely connected to cyclic symmetry. Indeed, for $d$ arbitrary, each of the $d$ sets

$$
E_{b}:=\left\{X^{a} Z^{b}: a=0,1, \ldots, d-1\right\} \quad b=0,1, \ldots, d-1
$$

is associated with an irreducible representation of the cyclic group $C_{d}$. More precisely, the one-dimensional irreducible representation of $C_{d}$ associated with $E_{b}$ is obtained by listing the nonzero matrix elements of any matrix of $E_{b}$, column by column from left to right. In this way, we obtain the $d$ irreducible representations of $C_{d}$. This relationship between $d$-dimensional Pauli matrices and irreducible representations of $C_{d}$ are clearly emphasized by the examples given above for $d=2,3$ and 4 .

\subsection{Pauli basis for the unitary group}

Two consequences follow from (114). (i) The Hilbert-Schmidt relation (114) in the Hilbert space $\mathbb{C}^{d^{2}}$ shows that the $d^{2}$ operators $u_{a b}$ are pairwise orthogonal operators. Thus, they can serve as a basis for developing any operator acting on $\mathcal{E}(d)$. (ii) The commutator in (113) defines the Lie bracket of a $d^{2}$-dimensional Lie algebra generated by the set $\left\{u_{a b}: a, b=0,1, \ldots, d-1\right\}$. This algebra can be identified to the Lie algebra $u(d)$ of the 
unitary group $U(d)$. The subset $\left\{u_{a b}: a, b=0,1, \ldots, d-1\right\} \backslash\left\{u_{00}\right\}$ then spans the Lie algebra $s u(d)$ of the special unitary group $S U(d)$. In other words, the Weyl pair $(X, Z)$, consisting of the generalized Pauli matrices $X$ and $Z$ in dimension $d$, form an integrity basis for $u(d)$. More specifically, the two following results hold.

Result 11. The set $\left\{X^{a} Z^{b}: a, b=0,1, \ldots, d-1\right\}$ forms a basis for the Lie algebra $u(d)$ of the unitary group $U(d)$. The Lie brackets of $u(d)$ in such a basis (denoted as the Pauli basis) are

$$
\left[X^{a} Z^{b}, X^{e} Z^{f}\right]_{-}=\sum_{i=0}^{d-1} \sum_{j=0}^{d-1}(a b, e f ; i j) X^{i} Z^{j}
$$

with the structure constants

$$
(a b, e f ; i j)=\delta(i, a \oplus e) \delta(j, b \oplus f)\left(q^{-b e}-q^{-a f}\right)
$$

where $a, b, e, f, i, j \in \mathbb{Z}_{d}$. The structure constants $(a b$, ef; $i j)$ with $i=a \oplus e$ and $j=b \oplus f$ are cyclotomic polynomials associated with $d$. They vanish for af $\ominus$ be $=0$.

Result 12. For $d=p$, with $p$ a prime integer, the Lie algebra $s u(p)$ of the special unitary group $S U(p)$ can be decomposed into a direct sum of $p+1$ abelian subalgebras of dimension $p-1$, i.e.

$$
s u(p) \simeq v_{0} \uplus v_{1} \uplus \ldots \uplus v_{p}
$$

where each of the $p+1$ subalgebras $v_{0}, v_{1}, \ldots, v_{p}$ is a Cartan subalgebra generated by a set of $p-1$ commuting matrices. The various sets are

$$
\begin{aligned}
\mathcal{V}_{0} & :=\left\{X^{0} Z^{1}, X^{0} Z^{2}, X^{0} Z^{3}, \ldots, X^{0} Z^{p-2}, X^{0} Z^{p-1}\right\} \\
\mathcal{V}_{1} & :=\left\{X^{1} Z^{0}, X^{2} Z^{0}, X^{3} Z^{0}, \ldots, X^{p-2} Z^{0}, X^{p-1} Z^{0}\right\} \\
\mathcal{V}_{2} & :=\left\{X^{1} Z^{1}, X^{2} Z^{2}, X^{3} Z^{3}, \ldots, X^{p-2} Z^{p-2}, X^{p-1} Z^{p-1}\right\} \\
\mathcal{V}_{3} & :=\left\{X^{1} Z^{2}, X^{2} Z^{4}, X^{3} Z^{6}, \ldots, X^{p-2} Z^{p-4}, X^{p-1} Z^{p-2}\right\} \\
& : \\
\mathcal{V}_{p-1} & :=\left\{X^{1} Z^{p-2}, X^{2} Z^{p-4}, X^{3} Z^{p-6}, \ldots, X^{p-2} Z^{4}, X^{p-1} Z^{2}\right\} \\
\mathcal{V}_{p} & :=\left\{X^{1} Z^{p-1}, X^{2} Z^{p-2}, X^{3} Z^{p-3}, \ldots, X^{p-2} Z^{2}, X^{p-1} Z^{1}\right\}
\end{aligned}
$$

for $v_{0}, v_{1}, \ldots, v_{p}$, respectively. 
Example 8: $p=7 \Leftrightarrow j=3$. Equations (146)-(152) give

$$
\begin{aligned}
& \mathcal{V}_{0}=\{(01),(02),(03),(04),(05),(06)\} \\
& \mathcal{V}_{1}=\{(10),(20),(30),(40),(50),(60)\} \\
& \mathcal{V}_{2}=\{(11),(22),(33),(44),(55),(66)\} \\
& \mathcal{V}_{3}=\{(12),(24),(36),(41),(53),(65)\} \\
& \mathcal{V}_{4}=\{(13),(26),(32),(45),(51),(64)\} \\
& \mathcal{V}_{5}=\{(14),(21),(35),(42),(56),(63)\} \\
& \mathcal{V}_{6}=\{(15),(23),(31),(46),(54),(62)\} \\
& \mathcal{V}_{7}=\{(16),(25),(34),(43),(52),(61)\}
\end{aligned}
$$

where $(a b)$ is used as an abbreviation of $X^{a} Z^{b}$.

Result 12 can be extended to the case where $d=p^{e}$ with $p$ a prime integer and $e$ a positive integer: there exists a decomposition of $s u\left(p^{e}\right)$ into $p^{e}+1$ abelian subalgebras of dimension $p^{e}-1$. In order to make this point clear, we start with a counterexample.

Counterexample: $d=4 \Leftrightarrow j=3 / 2(\Rightarrow a, b=0,1,2,3)$. In this case, Result 11 is valid but Result 12 does not apply. Indeed, the 16 unitary operators $u_{a b}$ corresponding to

$$
a b=01,02,03,10,20,30,11,22,33,12,13,21,23,31,32,00
$$

are linearly independent and span the Lie algebra of $U(4)$ but they give only three disjoint sets, viz., $\{(01),(02),(03)\},\{(10),(20),(30)\}$ and $\{(11),(22),(33)\}$, containing each 3 commuting operators, where here again $(a b)$ stands for $X^{a} Z^{b}$. However, it is not possible to partition the set (161) in order to get a decomposition similar to (145). Nevertheless, it is possible to find another basis of $u(4)$ which can be partitioned in a way yielding a decompostion similar to (145). This can be achieved by working with tensorial products of the matrices $X^{a} Z^{b}$ corresponding to $p=2$. In this respect, let us consider the product $u_{a_{1} b_{1}} \otimes u_{a_{2} b_{2}}$, where $u_{a_{i} b_{i}}$ with $i=1,2$ are Pauli operators for $p=2$. Then, by using the abbreviation $\left(a_{1} b_{1} a_{2} b_{2}\right)$ for $u_{a_{1} b_{1}} \otimes u_{a_{2} b_{2}}$ or $X^{a_{1}} Z^{b_{1}} \otimes X^{a_{2}} Z^{b_{2}}$, it can be checked that the five disjoint sets

$$
\begin{aligned}
& \{(1011),(1101),(0110)\} \\
& \{(1110),(1001),(0111)\} \\
& \{(1010),(1000),(0010)\} \\
& \{(1111),(1100),(0011)\} \\
& \{(0101),(0100),(0001)\}
\end{aligned}
$$


consist each of 3 commuting unitary operators and that the Lie algebra $s u(4)$ is spanned by the union of the 5 sets. It is to be emphasized that the 15 operators (162)-(166) are underlaid by the geometry of the generalized quadrangle of order 2 [90]. In this geometry, the five sets given by (162)-(166) correspond to a spread of this quadrangle, i.e., to a set of 5 pairwise skew lines [90].

The considerations of the counterexample can be generalized to $d:=d_{1} d_{2} \ldots d_{e}$, e being an integer greater or equal to 2 . Let us define

$$
u_{A B}:=u_{a_{1} b_{1}} \otimes u_{a_{2} b_{2}} \otimes \ldots \otimes u_{a_{e} b_{e}} \quad A:=a_{1}, a_{2}, \ldots, a_{e} \quad B:=b_{1}, b_{2}, \ldots, b_{e}
$$

where $u_{a_{1} b_{1}}, u_{a_{2} b_{2}}, \ldots, u_{a_{e} b_{e}}$ are generalized Pauli operators corresponding to the dimensions $d_{1}, d_{2}, \ldots, d_{e}$ respectively. In addition, let $q_{1}, q_{2}, \ldots, q_{e}$ be the $q$-factor associated with $d_{1}, d_{2}, \ldots, d_{e}$ respectively $\left(q_{j}:=\exp \left(2 \pi i / d_{j}\right)\right)$. Then, Results 10,11 and 12 can be generalized as follows.

Result 13. The operators $u_{A B}$ are unitary and satisfy the orthogonality relation

$$
\operatorname{Tr}_{\mathcal{E}\left(d_{1} d_{2} \ldots d_{e}\right)}\left[\left(u_{A B}\right)^{\dagger} u_{A^{\prime} B^{\prime}}\right]=d_{1} d_{2} \ldots d_{e} \delta_{A, A^{\prime}} \delta_{B, B^{\prime}}
$$

where

$$
\delta_{A, A^{\prime}}:=\delta_{a_{1}, a_{1}^{\prime}} \delta_{a_{2}, a_{2}^{\prime}} \ldots \delta_{a_{e}, a_{e}^{\prime}} \quad \delta_{B, B^{\prime}}:=\delta_{b_{1}, b_{1}^{\prime}} \delta_{b_{2}, b_{2}^{\prime}} \ldots \delta_{b_{e}, b_{e}^{\prime}}
$$

The commutator $\left[u_{A B}, u_{A^{\prime} B^{\prime}}\right]_{-}$and the anti-commutator $\left[u_{A B}, u_{A^{\prime} B^{\prime}}\right]_{+}$of $u_{A B}$ and $u_{A^{\prime} B^{\prime}}$ are given by

$$
\left[u_{A B}, u_{A^{\prime} B^{\prime}}\right]_{\mp}=\left(\prod_{j=1}^{e} q_{j}^{-b_{j} a_{j}^{\prime}} \mp \prod_{j=1}^{e} q_{j}^{-a_{j} b_{j}^{\prime}}\right) u_{A^{\prime \prime} B^{\prime \prime}}
$$

with

$$
A^{\prime \prime}:=a_{1} \oplus a_{1}^{\prime}, a_{2} \oplus a_{2}^{\prime}, \ldots, a_{e} \oplus a_{e}^{\prime} \quad B^{\prime \prime}:=b_{1} \oplus b_{1}^{\prime}, b_{2} \oplus b_{2}^{\prime}, \ldots, b_{e} \oplus b_{e}^{\prime} .
$$

The set $\left\{u_{A B}: A, B \in \mathbb{Z}_{d_{1}} \otimes \mathbb{Z}_{d_{2}} \otimes \ldots \otimes \mathbb{Z}_{d_{e}}\right\}$ of the $d_{1}^{2} d_{2}^{2} \ldots d_{e}^{2}$ unitary operators $u_{A B}$ form a basis for the Lie algebra $u\left(d_{1} d_{2} \ldots d_{e}\right)$ of the group $U\left(d_{1} d_{2} \ldots d_{e}\right)$.

The operators $u_{A B}$ may be called generalized Dirac operators since the ordinary Dirac operators correspond to specific $u_{a_{1} b_{1}} \otimes u_{a_{2} b_{2}}$ for $d_{1}=d_{2}=2$.

In the special case where $d_{1}=d_{2}=\ldots=d_{e}=p$ with $p$ a prime integer (or equivalently $d=p^{e}$ ), we have $\left[u_{A B}, u_{A^{\prime} B^{\prime}}\right]_{-}=0$ if and only if

$$
\sum_{j=1}^{e} a_{j} b_{j}^{\prime} \ominus b_{j} a_{j}^{\prime}=0
$$


and $\left[u_{A B}, u_{A^{\prime} B^{\prime}}\right]_{+}=0$ if and only if

$$
\sum_{j=1}^{e} a_{j} b_{j}^{\prime} \ominus b_{j} a_{j}^{\prime}=\frac{1}{2} p
$$

so that there are vanishing anti-commutators only if $p=2$. The commutation relations given by (170)-(171) can be transcribed in terms of Lagrangian submodules [65, 91]. For $d=p^{e}$, there exists a decomposition of the set $\left\{u_{A B}: A, B \in \mathbb{Z}_{p}^{\otimes e}\right\} \backslash\{I\}$ that corresponds to a decomposition of the Lie algebra $s u\left(p^{e}\right)$ into $p^{e}+1$ abelian subalgebras of dimension $p^{e}-1[25,74,92,93,94,95]$.

\subsection{Generalized Pauli group}

Let us define the $d^{3}$ operators

$$
w_{a b c}:=q^{a} x^{b} z^{c}=q^{a} u_{b c} \quad a, b, c \in \mathbb{Z}_{d}
$$

The action of $w_{a b c}$ on the Hilbert space $\mathcal{E}(d)$ is described by

$$
w_{a b c}|k\rangle=q^{a+k c}|k \ominus b\rangle \Leftrightarrow w_{a b c}|j, m\rangle=q^{a+(j-m) c}|j, m \oplus b\rangle .
$$

The operators $w_{a b c}$ are unitary and satisfy

$$
\operatorname{Tr}_{\mathcal{E}(d)}\left[\left(w_{a b c}\right)^{\dagger} w_{a^{\prime} b^{\prime} c^{\prime}}\right]=q^{a^{\prime}-a} d \delta_{b, b^{\prime}} \delta_{c, c^{\prime}}
$$

which gives back (114) for $a=a^{\prime}=0$.

The product of the operators $w_{a b c}$ and $w_{a^{\prime} b^{\prime} c^{\prime}}$ reads

$$
w_{a b c} w_{a^{\prime} b^{\prime} c^{\prime}}=w_{a^{\prime \prime} b^{\prime \prime} c^{\prime \prime}} \quad a^{\prime \prime}=a \oplus a^{\prime} \ominus c b^{\prime} \quad b^{\prime \prime}=b \oplus b^{\prime} \quad c^{\prime \prime}=c \oplus c^{\prime} .
$$

The set $\left\{w_{a b c}: a, b, c \in \mathbb{Z}_{d}\right\}$ can be endowed with a group structure. In the detail, we have the following

Result 14. The set $\left\{w_{a b c}: a, b, c \in \mathbb{Z}_{d}\right\}$, endowed with the internal law (177), is a finite group of order $d^{3}$. This nonabelian group, noted $\Pi_{d}$ and called generalized Pauli group in $d$ dimensions, is nilpotent (hence solvable) with a nilpotency class equal to 2. The group $\Pi_{d}$ is isomorphic to a subgroup of $U(d)$ for d even or $S U(d)$ for $d$ odd. It has $d(d+1)-1$ conjugacy classes (d classes containing each 1 element and $d^{2}-1$ classes containing each d elements) and $d(d+1)-1$ classes of irreducible representations ( $d^{2}$ classes of dimension 1 and $d-1$ classes of dimension $d)$. 
A faithful three-dimensional representation of $\Pi_{d}$ is provided with the application

$$
\Pi_{d} \rightarrow G L\left(3, \mathbb{Z}_{d}\right): w_{a b c} \mapsto\left(\begin{array}{ccc}
1 & 0 & 0 \\
b & 1 & 0 \\
a & -c & 1
\end{array}\right)
$$

This is reminiscent of the Heisenberg-Weyl group [96, 97, 98, 99, 100, 101]. Indeed, the group $\Pi_{d}$ can be considered as a discretization $H W\left(\mathbb{Z}_{d}\right)$ of the Heisenberg-Weyl group $H W(\mathbb{R})$, a three-parameter Lie group. The Heisenberg-Weyl group $H W(\mathbb{R})$, also called the Heisenberg group or Weyl group, is at the root of quantum mechanics. It also plays an important role in symplectic geometry. The group $\Pi_{d}$ was discussed by Štovíček and Tolar [102] in connection with quantum mechanics in a discrete space-time, by Balian and Itzykson [73] in connection with finite quantum mechanics, by Patera and Zassenhaus [74] in connection with gradings of simple Lie algebras of type $A_{n-1}$, and by Kibler [25] in connection with Weyl pairs and the Heisenberg-Weyl group. Note that the discrete version $H W(\mathbb{Z})$ of $H W(\mathbb{R})$ was used for an analysis of the solutions of the Markoff equation [103]. Recently, the discrete version $H W\left[\mathbb{Z}_{p} \times\left(\mathbb{Q}_{p} / \mathbb{Z}_{p}\right)\right]$ was introduced for describing ( $p$-adic) quantum systems with positions in $\mathbb{Z}_{p}$ and momenta in $\mathbb{Q}_{p} / \mathbb{Z}_{p}[104]$.

As far as $H W\left(\mathbb{Z}_{d}\right)$ is concerned, it is to be observed that a Lie algebra $\pi_{d}$ can be associated with the finite group $\Pi_{d}$. This can be seen by considering the Frobenius algebra of $\Pi_{d}$ (see [105] for the definition of the Lie algebra associated with an arbitray finite group). Then, the Lie brackets of $\pi_{d}$ are

$$
\left[w_{a b c}, w_{a^{\prime} b^{\prime} c^{\prime}}\right]_{-}=w_{\alpha \beta \gamma}-w_{\alpha^{\prime} \beta^{\prime} \gamma^{\prime}}
$$

with

$$
\alpha=a \oplus a^{\prime} \ominus c b^{\prime} \quad \beta=b \oplus b^{\prime} \quad \gamma=c \oplus c^{\prime} \quad \alpha^{\prime}=\alpha \oplus c b^{\prime} \ominus b c^{\prime} \quad \beta^{\prime}=\beta \quad \gamma^{\prime}=\gamma
$$

The algebra $\pi_{d}$, of dimension $d^{3}$, is not semisimple. It can be decomposed as the direct sum

$$
\pi_{d} \simeq \biguplus_{1}^{d^{2}} u(1) \biguplus_{1}^{d-1} u(d)
$$

which contains $d^{2}$ Lie algebras isomorphic to $u(1)$ and $d-1$ Lie algebras isomorphic to $u(d)$. The Lie algebra $u(d)$ spanned by the set $\left\{u_{a b}: a, b \in \mathbb{Z}_{d}\right\}$ is one of the subalgebras of $\pi_{d}$.

The group $\Pi_{d}$ (noted $P_{d}$ in [25]) should not be confused with the Pauli group $\mathcal{P}_{n}$ on $n$ qubits spanned by $n$-fold tensor products of $i \sigma_{0} \equiv i I_{2}, \sigma_{x}$ and $\sigma_{z}$ used in quantum information and quantum computation [106, 107]. The Pauli group $\mathcal{P}_{n}$ has $4^{n+1}$ 
elements. It is used as an error group in quantum computing. The normaliser of $\mathcal{P}_{n}$ in $S U\left(2^{n}\right)$, known as the Clifford (or Jacobi) group $C l i_{n}$ on $n$ qubits, a group of order $2^{n^{2}+2 n+3} \prod_{j=1}^{n}\left(4^{j}-1\right)$, is of great interest in the context of quantum corrector codes [41, 108, 109, 110, 111, 112, 113]. In addition to $C l i_{n}$, proper sugroups of $C l i_{n}$ having $\mathcal{P}_{n}$ as an invariant subgroup are relevant for displaying quantum coherence [114]. The distinction bewteen $\mathcal{P}_{n}$ and $\Pi_{d}$ can be clarified by the example below which shows that $\Pi_{2}$ is not isomorphic to $\mathcal{P}_{1}$. In a parallel way, it can be proved that the groups $\Pi_{4}$ and $\mathcal{P}_{2}$ (both of order 64) are distinct.

Example 9: $d=2$. The simplest example of $\Pi_{d}$ occurs for $d=2$. The group $\Pi_{2}$ has 8 elements $( \pm I, \pm x, \pm y:= \pm x z, \pm z)$ and is isomorphic to the dihedral group $D_{4}$. It can be partitioned into 5 conjugation classes $(\{I\},\{-I\},\{x,-x\},\{y,-y\},\{z,-z\})$ and possesses 5 inequivalent irreducible representations (of dimensions 1, 1, 1, 1 and 2). The two-dimensional irreducible representation corresponds to

$$
\pm I \mapsto \pm \sigma_{0} \quad \pm x \mapsto \pm \sigma_{x} \quad \pm y \mapsto \mp i \sigma_{y} \quad \pm z \mapsto \pm \sigma_{z}
$$

in terms of the Pauli matrices $\sigma_{\lambda}$ with $\lambda=0, x, y, z$. The elements $e_{1}:=x, e_{2}:=y$ and $e_{3}:=z$ of $\Pi_{2}$ span the four-dimensional algebra $A(1,-1,0) \equiv \mathbb{N}_{1}$, the algebra of hyperbolic quaternions (with $e_{1}^{2}=-e_{2}^{2}=e_{3}^{2}=1$ instead of $e_{1}^{2}=e_{2}^{2}=e_{3}^{2}=-1$ as for usual quaternions). This associative and noncommutative algebra is a singular division algebra. The algebra $\mathbb{N}_{1}$ turns out to be a particular Cayley-Dickson algebra $A\left(c_{1}, c_{2}, c_{3}\right)$ [115]. Going back to $\Pi_{2}$, we see that not all the subgroups of $\Pi_{2}$ are invariant. The group $\Pi_{2}$ is isomorphic to the group of hyperbolic quaternions rather than to the group $Q$ of ordinary quaternions for which all subgroups are invariant (the group $Q$ can be realized with the help of the matrices $\pm \sigma_{0}, \pm i \sigma_{x}, \pm i \sigma_{y}, \pm i \sigma_{z}$ ). Like $Q$, the group $\Pi_{2}$ is ambivalent and simply reducible in the terminology of Wigner [2]. Indeed, $\Pi_{2}$ is the sole generalized Pauli group that is ambivalent.

To end up with this example, let us examine the connection between $\Pi_{2}$ and the 1 qubit Pauli group $\mathcal{P}_{1}$. The group $\mathcal{P}_{1}$ has 16 elements $\left( \pm \sigma_{\lambda}, \pm i \sigma_{\lambda}\right.$ with $\left.\lambda=0, x, y, z\right)$. Obviously, $\Pi_{2}$ is a subgroup of index 2 (necessarily invariant) of $\mathcal{P}_{1}$. The group $\mathcal{P}_{1}$ can be considered as a double group of $\Pi_{2}$ or $Q$ in the sense that $\mathcal{P}_{1}$ coincides with $\Pi_{2} \bigcup i \Pi_{2} \equiv Q \bigcup i Q$ in terms of sets. Therefore, the group table of $\mathcal{P}_{1}$ easily follows from the one of $\Pi_{2}$ or $Q$. As a result, the numbers of conjugation classes and irreducible representation classes are doubled when passing from $\Pi_{2}$ or $Q$ to $\mathcal{P}_{1}$. 


\section{Closing remarks}

Starting from a nonstandard approach to angular momentum and its transcription in terms of representation of $S U(2)$, we derived (in an original and unified way) some results about unitary operator bases and their connection to unitary groups, Pauli groups and quadratic sums. These results (either known or formulated in a new way) shed some light on the importance of the polar decomposition of $s u(2)$ and cyclic groups for the study of unitary operator bases and their relationship with unitary groups and Pauli groups. In particular, the latter decomposition and the quadratic discrete Fourier transform introduced in this work make it possible to generate in prime dimension a complete set of MUBs given by a single formula (Eq. (20)). From the point of view of the representation theory, it would be interesting to find realisation on the state vectors (20) on the sphere $S^{2}$ thus establishing a contact between the $\left\{j^{2}, v_{0 a}\right\}$ scheme and special functions.

To close this paper, let us be mention two works dealing with à la Schwinger unitary operator bases in an angular momentum scheme. In Ref. [116], unitary operator bases and standard (discrete) quantum Fourier transforms in an angular momentum framework proved to be useful for spin tunneling. In addition, $d$-dimensional generalized Pauli matrices applied to modified Bessel functions were considered in an angular momentum approach with $j=(d-1) / 2 \rightarrow \infty$ [117].

\section{Appendix A: A polar decomposition of $s u(2)$}

In addition to the operator $v_{r a}$, a second linear operator is necessary to define a polar decomposition of $S U(2)$. Let us introduce the Hermitian operator $h$ through

$$
h:=\sum_{m=-j}^{j} \sqrt{(j+m)(j-m+1)}|j, m\rangle\langle j, m| .
$$

Then, it is a simple matter of calculation to show that the three operators

$$
j_{+}:=h v_{r a} \quad j_{-}:=\left(v_{r a}\right)^{\dagger} h \quad j_{z}:=\frac{1}{2}\left[h^{2}-\left(v_{r a}\right)^{\dagger} h^{2} v_{r a}\right]
$$

satisfy the ladder equations

$$
\begin{aligned}
& j_{+}|j, m\rangle=q^{+(j-m+s-1 / 2) a} \sqrt{(j-m)(j+m+1)}|j, m+1\rangle \\
& j_{-}|j, m\rangle=q^{-(j-m+s+1 / 2) a} \sqrt{(j+m)(j-m+1)}|j, m-1\rangle
\end{aligned}
$$

and the eigenvalue equation

$$
j_{z}|j, m\rangle=m|j, m\rangle
$$


where $s=1 / 2$. Therefore, the operators $j_{+}, j_{-}$and $j_{z}$ satisfy the commutation relations

$$
\left[j_{z}, j_{+}\right]_{-}=+j_{+} \quad\left[j_{z}, j_{-}\right]_{-}=-j_{-} \quad\left[j_{+}, j_{-}\right]_{-}=2 j_{z}
$$

and thus span the Lie algebra of $S U(2)$.

The latter result does not depend on the parameters $r$ and $a$. However, the action of $j_{+}$and $j_{-}$on $|j, m\rangle$ on the space $\mathcal{E}(2 j+1)$ depends on $a$; the usual Condon and Shortley phase convention used in spectroscopy corresponds to $a=0$. The writing of the ladder operators $j_{+}$and $j_{-}$in terms of $h$ and $v_{r a}$ constitutes a two-parameter polar decomposition of the Lie algebra of $S U(2)$. This decomposition is an alternative to the polar decompositions obtained independently in [24, 118, 119, 120, 121].

\subsection{Appendix B: A quon approach to $s u(2)$}

Following [122], we define a quon algebra or $q$-deformed oscillator algebra for $q$ a root of unity. The three operators $a_{-}, a_{+}$and $N_{a}$ such that

$$
a_{-} a_{+}-q a_{+} a_{-}=I \quad\left[N_{a}, a_{ \pm}\right]_{-}= \pm a_{ \pm} \quad\left(a_{ \pm}\right)^{k}=0 \quad N_{a}^{\dagger}=N_{a}
$$

where

$$
q:=\exp \left(\frac{2 \pi i}{k}\right) \quad k \in \mathbb{N} \backslash\{0,1\}
$$

define a quon algebra or $q$-deformed oscillator algebra denoted as $A_{q}\left(a_{-}, a_{+}, N_{a}\right)$. The operators $a_{-}$and $a_{+}$are referred to as quon operators. The operators $a_{-}, a_{+}$and $N_{a}$ are called annihilation, creation and number operators, respectively.

Let us consider two commuting quon algebras $A_{q}\left(a_{-}, a_{+}, N_{a}\right) \equiv A_{q}(a)$ with $a=x, y$ corresponding to the same value of the deformation parameter $q$. Their generators satisfy equations (189) and (190) with $a=x, y$ and $[X, Y]_{-}=0$ for any $X$ in $A_{q}(x)$ and any $Y$ in $A_{q}(y)$. Then, let us look for Hilbertian representations of $A_{q}(x)$ and $A_{q}(y)$ on the $k$-dimensional Hilbert spaces $\mathcal{F}(x)$ and $\mathcal{F}(y)$ spanned by the orthonormal bases $\left.\left\{\mid n_{1}\right): n_{1}=0,1, \ldots, k-1\right\}$ and $\left.\left\{\mid n_{2}\right): n_{2}=0,1, \ldots, k-1\right\}$, respectively. We easily obtain the representations defined by

$$
\begin{aligned}
& \left.\left.\left.x_{+} \mid n_{1}\right)=\mid n_{1}+1\right) \quad x_{+} \mid k-1\right)=0 \\
& \left.\left.\left.x_{-} \mid n_{1}\right)=\left[n_{1}\right]_{q} \mid n_{1}-1\right) \quad x_{-} \mid 0\right)=0 \\
& \left.\left.N_{x} \mid n_{1}\right)=n_{1} \mid n_{1}\right)
\end{aligned}
$$


and

$$
\begin{aligned}
& \left.\left.\left.y_{+} \mid n_{2}\right)=\left[n_{2}+1\right]_{q} \mid n_{2}+1\right) \quad y_{+} \mid k-1\right)=0 \\
& \left.\left.\left.y_{-} \mid n_{2}\right)=\mid n_{2}-1\right) \quad y_{-} \mid 0\right)=0 \\
& \left.\left.N_{y} \mid n_{2}\right)=n_{2} \mid n_{2}\right)
\end{aligned}
$$

for $A_{q}(x)$ and $A_{q}(y)$, respectively.

The cornerstone of this approach is to define the two linear operators

$$
h:=\sqrt{N_{x}\left(N_{y}+1\right)} \quad v_{r a}:=s_{x} s_{y}
$$

with

$$
\begin{aligned}
& s_{x}:=q^{a\left(N_{x}+N_{y}\right) / 2} x_{+}+e^{i \phi_{r} / 2} \frac{1}{[k-1]_{q} !}\left(x_{-}\right)^{k-1} \\
& s_{y}:=y_{-} q^{-a\left(N_{x}-N_{y}\right) / 2}+e^{i \phi_{r} / 2} \frac{1}{[k-1]_{q} !}\left(y_{+}\right)^{k-1}
\end{aligned}
$$

where

$$
a \in \mathbb{R} \quad \phi_{r}=\pi(k-1) r \quad r \in \mathbb{R} .
$$

The operators $h$ and $v_{r a}$ act on the states

$$
\left.\left.\left.\mid n_{1}, n_{2}\right):=\mid n_{1}\right) \otimes \mid n_{2}\right)
$$

of the $k^{2}$-dimensional space $\mathcal{F}_{k}:=\mathcal{F}(x) \otimes \mathcal{F}(y)$. It is immediate to show that the action of $h$ and $v_{r a}$ on $\mathcal{F}_{k}$ is given by

$$
\left.\left.h \mid n_{1}, n_{2}\right)=\sqrt{n_{1}\left(n_{2}+1\right)} \mid n_{1}, n_{2}\right) \quad n_{i}=0,1,2, \cdots, k-1 \quad i=1,2
$$

and

$$
\begin{gathered}
\left.\left.v_{r a} \mid n_{1}, n_{2}\right)=q^{a n_{2}} \mid n_{1}+1, n_{2}-1\right) \quad n_{1} \neq k-1 \quad n_{2} \neq 0 \\
\left.\left.v_{r a} \mid k-1, n_{2}\right)=e^{i \phi_{r} / 2} q^{-a\left(k-1-n_{2}\right) / 2} \mid 0, n_{2}-1\right) \quad n_{2} \neq 0 \\
\left.\left.v_{r a} \mid n_{1}, 0\right)=e^{i \phi_{r} / 2} q^{a\left(k+n_{1}\right) / 2} \mid n_{1}+1, k-1\right) \quad n_{1} \neq k-1 \\
\left.\left.v_{r a} \mid k-1,0\right)=e^{i \phi_{r}} \mid 0, k-1\right) .
\end{gathered}
$$


The operators $h$ and $v_{r a}$ satisfy interesting properties: the operator $h$ is Hermitian and the operator $v_{r a}$ is unitary.

We now adapt the trick used by Schwinger [123] in his approach to angular momentum via a coupled pair of harmonic oscillators. This can be done by introducing two new quantum numbers $J$ and $M$ defined by

$$
\left.\left.J:=\frac{1}{2}\left(n_{1}+n_{2}\right) \quad M:=\frac{1}{2}\left(n_{1}-n_{2}\right) \Rightarrow|J M\rangle:=\mid J+M, J-M\right)=\mid n_{1}, n_{2}\right) .
$$

Note that

$$
j:=\frac{1}{2}(k-1)
$$

is an admissible value for $J$. Then, let us consider the $k$-dimensional subspace $\epsilon(j)$ of the $k^{2}$-dimensional space $\mathcal{F}(x) \otimes \mathcal{F}(y)$ spanned by the basis $\{|j, m\rangle: m=j, j-1, \ldots,-j\}$. We guess that $\epsilon(j)$ is a space of constant angular momentum $j$. As a matter of fact, we can check that $\epsilon(j)$ is stable under $h$ and $v_{r a}$. In fact, the action of the operators $h$ and $v_{r a}$ on the subspace $\varepsilon(j)$ of $\mathcal{F}_{k}$ can be described by

$$
h|j, m\rangle=\sqrt{(j+m)(j-m+1)}|j, m\rangle
$$

and

$$
v_{r a}|j, m\rangle=\delta_{m, j} e^{i 2 \pi j r}|j,-j\rangle+\left(1-\delta_{m, j}\right) q^{(j-m) a}|j, m+1\rangle
$$

in agreement with Eq. (183) and with the master equation (6).

\section{References}

[1] Biedenharn L C and Louck J D 1985 Angular momentum in quantum physics Encyclopedia of Mathematics and Its Applications Vol 8 ed G-C Rota (Cambridge: Cambridge University Press)

[2] Wigner E P 1941 Am. J. Math. 6357

Wigner E P 1965 On the matrices which reduce the Kronecker products of representations of S.R. groups Quantum Theory of Angular Momemtum eds L C Biedenharn and $\mathrm{H}$ van Dam (New York: Academic Press)

[3] Racah G 1942 Phys. Rev. 62438

Racah G 1949 Phys. Rev. 761352

Racah G 1961 Group theory and spectroscopy Report CERN 61-8 (Geneva: CERN) 
[4] Biedenharn L C and Louck J D 1985 The Racah-Wigner algebra in quantum theory Encyclopedia of Mathematics and Its Applications Vol 9 ed G-C Rota (Cambridge: Cambridge University Press)

[5] Sharp W T 1960 Report AECL-1098 (Chalk River, Ontario: Atomic Energy of Canada Ltd)

Derome J R and Sharp W T 1965 J. Math. Phys. 61584

[6] Cottingham W N and Greenwood D A 2007 An Introduction to the Standard Model of Particle Physics (Cambridge: Cambridge University Press)

[7] Sviridov D T and Smirnov Yu F 1977 Teoriya Opticheskikh Spektrov Perekhodnykh Metallov (Moscow: Izd. Nauka)

[8] Tang Au-chin, Sun Chia-chung, Kiang Yuan-sun, Deng Zung-hau, Liu Jo-Chuang, Chang Chian-er, Yan Guo-sen, Goo Zien and Tai Shu-san 1979 Theoretical Method of the Ligand Field Theory (Peking: Science Press)

[9] M.R. Kibler 1979 Finite symmetry adaptation in spectroscopy Recent Advances in Group Theory and Their Application to Spectroscopy ed J C Donini (New York: Plenum Press)

[10] Ma E 2007 Preprint $\operatorname{arXiv:0705.0327v4~[hep-ph]~}$

[11] Bonatsos D, Raychev P P, Roussev R P and Smirnov Yu F 1990 Chem. Phys. Lett. 175300

Bonatsos D, Drenska S B, Raychev P P, Roussev R P and Smirnov Yu F $1991 J$. Phys. G: Nucl. Part. Phys. 17 L67

[12] Smirnov Yu F and Kibler M R 1993 Some aspects of $q$-boson calculus Symmetries in Science VI: From the Rotation Group to Quantum Algebras ed B Gruber (New York: Plenum Press)

Kibler M, Campigotto C and Smirnov Yu F 1994 Recursion relations for ClebschGordan coefficients of $U_{q}\left(s u_{2}\right)$ and $U_{q}\left(s u_{1,1}\right)$ Symmetry Methods in Physics eds A N Sissakian, G S Pogosyan and S I Vinitsky (Dubna: Joint Institute for Nuclear Research)

Kibler M R, Asherova R M and Smirnov Yu F 1995 Some aspects of $q$ - and $q p$ boson calculus Symmetries in Science VIII ed B Gruber (New York: Plenum Press)

[13] Kibler M R 1999 On the Wigner-Racah algebra of the group $S U_{2}$ in a non-standard basis Symmetry and Structural Properties of Condensed Matter eds T Lulek, B Lulek and A Wal (Singapore: World Scientific)

Kibler M and Daoud M 2001 Recent Res. Devel. Quantum Chem. 291

Kibler M R 2005 Collect. Czech. Chem. Commun. 70771 
[14] Albouy O and Kibler M R 2007 SIGMA 3 article 076

[15] Melvin M A 1956 Rev. Mod. Phys. 2818

[16] Altmann S L and Cracknell A P 1965 Rev. Mod. Phys. 3719

Altmann S L and Bradley C J 1965 Rev. Mod. Phys. 3733

[17] Michelot F and Moret-Bailly J 1975 J. Phys. (Paris) 36451

Champion J P, Pierre G, Michelot F and Moret-Bailly J 1977 Can. J. Phys. 55512

Champion J P 1977 Can. J. Phys. 551802

[18] Kibler M 1969 Int. J. Quantum Chem. 3795

Kibler M and Grenet G 1986 Int. J. Quantum Chem. 29485

Kibler M and Gâcon J C 1989 Croat. Chem. Acta 62783

[19] Moret-Bailly J 1965 J. Mol. Spectrosc. 15344

[20] Kibler M 1968 J. Mol. Spectrosc. 26111

Kibler M 1969 C. R. Acad. Sci. (Paris) B 2681221

[21] Patera J and Winternitz P 1973 J. Math. Phys. 141130

Patera J and Winternitz P 1976 J. Chem. Phys. 652725

[22] Michel L 1977 Invariants polynomiaux des groupes de symétrie moléculaire et cristallographique Group Theoretical Methods in Physics eds R T Sharp and B Kolman (New York: Academic Press)

[23] Grenet G, Zhilinskii B I and Kibler M 1980 Vestnik Moskovsk. Univ., ser. Khim. 21 244

[24] Vourdas A 2004 Rep. Prog. Phys. 67267

[25] Kibler M R 2008 J. Phys. A: Math. Theor. 41375302

[26] Diţă P 2005 J. Phys. A: Math. Gen. 375355

[27] Bengtsson I, Bruzda W, Ericsson Å, Larsson J-Å, Tadej W and Życkowski K 2007 J. Math. Phys. 48052106

[28] Brierley S and Weigert S 2009 Preprint arXiv:0901.4051v1 [quant-ph]

[29] Tadej W and Życzkowski K 2005 Preprint quant-ph/0512154

Tadej W and Życzkowski K 2006 Open Sys. Info. Dynamics 13133

Tadej W and Życzkowski K 2009 On-line catalogue of known Hadamard matrices http://chaos.if.uj.edu.pl/ karol/hadamard/ 
[30] Kibler M R 2006 Int. J. Mod. Phys. B 201792

Kibler M R and Planat M 2006 Int. J. Mod. Phys. B 201802

[31] Berndt B C and Evans R J 1981 Bull. Am. Math. Soc. 5107

Berndt B C, Evans R J and Williams K S 1998 Gauss and Jacobi Sums (New York: Wiley)

[32] Hannay J H and Berry M V 1980 Physica D 1267

[33] Matsutani S and Ônishi Y 2003 Found. Phys. Lett. 16325

[34] Rosu H C, Treviño J P, Cabrera H and Murguía J S 2006 Int. J. Mod. Phys. B 20 1860

[35] Merkel W, Crasser O, Haug F, Lutz E, Mack H, Freyberger M, Schleich W P, Averbukh I, Bienert M, Girard B, Maier H and Paulus G G 2006 Int. J. Mod. Phys. B 201893

[36] Delsarte P, Goethals J M and Seidel J J 1975 Philips Res. Repts. 3091

[37] Ivanović I D 1981 J. Phys. A: Math. Gen. 143241

[38] Wootters W K 1986 Found. Phys. 16391

Wootters W K 1987 Ann. Phys. (N.Y.) 1761

Wootters W K and Fields B D 1989 Ann. Phys. (N Y) 191363

Wootters W K 2006 Found. Phys. 36112

[39] Calderbank A R, Cameron P J, Kantor W M and Seidel J J 1997 Proc. London Math. Soc. 75436

[40] Zauner G 1999 Quantendesigns: Grundzüge einer nichtcommutativen Designtheorie Diploma Thesis University of Wien

[41] Grassl M 2005 On SIC-POVMs and MUBs in dimension 6, Proc. ERATO Conference on Quantum Information Science (EQIS 2004) ed J Gruska (Tokyo)

Grassl M 2005 Elec. Notes Discrete Math. 20151

[42] Brierley S and Weigert S 2008 Phys. Rev. A 78042312

[43] Heath R W, Strohmer T and Paulraj A J 2006 IEEE Transactions on Information Theory $\mathbf{5 2} 1217$

[44] Cerf N J, Bourennane M, Karlsson A and Gisin N 2002 Phys. Rev. Lett. 88127902

[45] Aharonov Y and Englert B-G 2001 Z. Naturforsch. 56a 16

Englert B-G and and Aharonov Y 2001 Phys. Lett. A 2841 
[46] Aravind P K 2003 Z. Naturforsch. 58a 2212

[47] Hayashi A, Horibe M and Hashimoto T 2005 Phys. Rev. A 71052331

[48] Paz J P, Roncaglia A J and Saraceno M 2005 Phys. Rev. A 72012309

[49] Wocjan P and Beth T 2005 Quantum Inf. Comput. 593

[50] Durt T 2006 Int. J. Mod. Phys. B 201742

[51] Svetlichny G 2007 Preprint arXiv:0708.3079 [quant-ph]

[52] Tolar J and Chadzitaskos G 2009 Feynman's path integral and mutually unbiased bases (submitted to J. Phys. A: Math. Theor.)

[53] Weigert S and Wilkinson M 2008 Phys. Rev. A 78020303

[54] Bandyopadhyay S, Boykin P O, Roychowdhury V and Vatan F 2002 Algorithmica 34512

[55] Lawrence J, Brukner Č and Zeilinger A 2002 Phys. Rev. A 65032320

Lawrence J 2004 Phys. Rev. A 70012302

[56] Chaturvedi S 2002 Phys. Rev. A 65044301

[57] Vlasov A Yu 2003 Preprint arXiv:0302064 [quant-ph]

[58] Klappenecker A and Rötteler M 2004 Lect. Notes Comput. Sci. 2948262

[59] Gibbons K S, Hoffman M J, Wootters W K 2004 Phys. Rev. A 70062101

[60] Pittenger A O and Rubin M H 2004 Linear Algebr. Appl. 390255

Pittenger A O and Rubin M H 2005 J. Phys. A: Math. Gen. 386005

[61] Durt T 2005 J. Phys. A: Math. Gen. 385267

[62] Archer C 2005 J. Math. Phys. 46022106

[63] Aschbacher M, Childs A M and Wocjan P 2007 J. Algebr. Comb. 25111

[64] Šulc P and Tolar J 2007 J. Phys. A: Math. Theor. 4015099

[65] Albouy O 2009 Discrete algebra and geometry applied to the Pauli group and mutually unbiased bases in quantum information theory Thesis Université de Lyon

[66] Albouy O 2009 Determinantal measure for pure states entanglement (submitted to J. Phys. A: Math. Theor.)

[67] Havlicek H and Saniga M 2007 J. Phys. A: Math. Theor. 40 F943

Havlicek H and Saniga M 2008 J. Phys. A: Math. Theor. 41015302 
[68] Planat M and Baboin A-C 2007 J. Phys. A: Math. Theor. 40 F1005

[69] Weyl H 1931 The Theory of Groups and Quantum Mechanics (New York: Dover Publications)

[70] Schwinger J 1960 Proc. Nat. Acad. Sci. USA 46570

[71] Sylvester J J 1883 C. R. Acad. Sci. (Paris) XCVII 1336

[72] Morris A O 1967 Quart. J. Math. 187

Morris A O 1968 Quart. J. Math. 19289

[73] Balian R and Itzykson C 1986 C. R. Acad. Sci. (Paris) 303773

[74] Patera J and Zassenhaus H 1988 J. Math. Phys. 29665

[75] Galetti D and De Toledo Piza A F R 1988 Physica A 149267

[76] Knill E 1996 Preprint quant-ph/9608048

[77] Gottesman D 1999 Chaos, Solitons and Fractals 101749

[78] Pittenger A O and Rubin M H 2000 Phys. Rev. A 62032313

[79] Gottesman D, Kitaev A and Preskill J 2001 Phys. Rev. A 64012310

[80] Bartlett S D, de Guise H and Sanders B C 2002 Phys. Rev. A 65052316

[81] Klimov A B, Sánchez-Soto L L and de Guise H 2005 J. Phys. A: Math. Gen. 38 2747

Klimov A B, Sánchez-Soto L L and de Guise H 2005 J. Opt. B: Quantum Semiclass. Opt. 7283

Sánchez-Soto L L, Klimov A B and de Guise H 2006 Int. J. Mod. Phys. B 201877

[82] Romero J L, Björk G, Klimov A B and Sánchez-Soto L L 2005 Phys. Rev. A 72 062310

[83] Ne'eman Y 1961 Nucl. Phys. 26222

[84] Gell-Mann M 1962 Phys. Rev. 1251067

[85] Okubo S 1962 Prog. Theor. Phys. (Kyoto) 27949

[86] Glashow S L, Iliopoulos J and Maiani L 1970 Phys. Rev. D 21285

[87] Georgi H and Glashow S L 1974 Phys. Rev. Lett. 32438

[88] Moffat J W 1975 Phys. Rev. D 12288 
[89] de Azcárraga J A and Macfarlane A J 2001 Int. J. Mod. Phys. A 161377

[90] Planat M, Saniga M and Kibler M R 2006 SIGMA 2 article 066

Saniga M, Planat M, Pracna P and Havlicek H 2007 SIGMA 3 article 075

Planat M, Baboin A-C and Saniga M 2008 Int. J. Theor. Phys. 471127

Planat M and Saniga M 2008 Quantum Inf. Comput. 80127

Saniga M, Planat M and Pracna P 2008 Theor. and Math. Phys. 155463

[91] Albouy O 2009 J. Phys. A: Math. Theor. 42072001

[92] Kostrikin A I, Kostrikin I A and Ufnarovskiǔ V A 1981 Proc. Steklov Inst. Math. 158105

Kostrikin A I, Kostrikin I A and Ufnarovskiŭ V A 1981 Sov. Math. Dokl. 24292

Kostrikin A I, Kostrikin I A and Ufnarovskiı̌ V A 1982 Sov. Math. Dokl. 252327

[93] Kostrikin A I and Tiep P H 1994 Expositions in mathematics 15

[94] Boykin P O, Sitharam M, Tiep P H and Wocjan P 2007 Quantum Inf. Comput. 7 371

[95] Bengtsson I 2006 Preprint quant-ph/0610216

[96] Wolf K B and García A 1972 Rev. Mex. Física 21191

[97] Wolf K B 1975 The Heisenberg-Weyl ring in quantum mechanics Group theory and its applications Vol. III ed E M Loebl (New York: Academic Press)

[98] García-Bullé M, Lassner W and Wolf K B 1986 J. Math. Phys. 2729

[99] Atakishiyev N M, Pogosyan G S and Wolf K B 2003 Int. J. Mod. Phys. A 18317

[100] Terras A 1996 Experimental Math. 515

Terras A 1999 Fourier Analysis on Finite Groups and Applications (Cambridge: Cambridge University Press)

[101] Howe R 2005 Indag. Mathem., N.S. 16553

[102] Šťovíček P and Tolar J 1984 Rep. Math. Phys. 20157

[103] Perrine S 2009 De Frobenius à Riedel : analyse des solutions de l'équation de Markoff Unpublished manuscript

[104] Vourdas A 2008 J. Phys. A: Math. Theor. 41455303

[105] Gamba A 1969 J. Math. Phys. 10872 
[106] Nielsen M A and Chuang I L 2000 Quantum computation and quantum information (Cambridge: Cambridge University Press)

[107] Jozsa R 2005 Preprint arXiv:0508124v2 [quant-ph]

[108] Gottesman D 1997 Preprint arXiv:9705052 [quant-ph]

[109] Calderbank A R, Rains E M, Schor P W and Sloane N J A 1998 IEEE Trans. Inform. Theory 441369

[110] Clark S, Jozsa R and Linden N 2008 Quant. Inform. Comp. 8106

[111] Appleby D M 2005 J. Math. Phys. 46052107

[112] Flammia S T 2006 J. Phys. A: Math. Gen. 3913483

[113] Cormick C, Galvão E F, Gottesman D, Paz J P and Pittenger A O 2006 Phys. Rev. A 73012301

[114] Planat M and Jorrand Ph 2008 J. Phys. A: Math. Theor. 41182001

[115] Lambert D and Kibler M 1988 J. Phys. A: Math. Gen. 21307

[116] Marchiolli M A, Silva E C and Galetti D 2009 Phys. Rev. A to appear

[117] Fujii K 2007 Int. G. Geom. Meth. Mod. Phys. 41205

[118] Lévy-Leblond J-M 1973 Rev. Mex. Física 2215

[119] Vourdas A 1990 Phys. Rev. A 411653

[120] Chaichian M and Ellinas D 1990 J. Phys. A: Math. Gen. 23 L291

[121] Ellinas D 1991 J. Math. Phys. 32135

[122] Daoud M, Hassouni Y and Kibler M 1998 The $k$-fermions as objects interpolating between fermions and bosons Symmetries in Science X eds B Gruber and M Ramek (New York: Plenum Press)

Daoud M, Hassouni Y and Kibler M 1998 Phys. Atom. Nuclei 611821

[123] Schwinger J 1965 On angular momentum Quantum theory of angular momemtum eds L C Biedenharn and H van Dam (New York: Academic Press) 